Submitted to ApJ on November 13, 2017; IN REVISEd FORM ON JANuARY 4, 2018; ACCEPTED ON JANUARY 6, 2018

Preprint typeset using $\mathrm{LATEX}_{\mathrm{E}}$ style emulateapj v. 12/16/11

\title{
THE EXTRAORDINARY OUTBURST IN THE MASSIVE PROTOSTELLAR SYSTEM NGC6334I-MM1: EMERGENCE OF STRONG 6.7 GHZ METHANOL MASERS
}

\author{
T. R. Hunter ${ }^{1}$, C. L. Brogan ${ }^{1}$, G. C. Macleod ${ }^{2}$, C. J. Cyganowski ${ }^{3}$, J. O. Chibueze ${ }^{4,5,6}$, R. Friesen $^{1,7}$, T. Hirota $^{8}$, \\ D. P. $\operatorname{Smits}^{9}$, C. J. ChandleR ${ }^{10}$, R. Indebetouw ${ }^{1,11}$ \\ submitted to ApJ on November 13, 2017; in revised form on January 4, 2018; accepted on January 6, 2018
}

\begin{abstract}
We report the first sub-arcsecond VLA imaging of $6 \mathrm{GHz}$ continuum, methanol maser, and excitedstate hydroxyl maser emission toward the massive protostellar cluster NGC6334I following the recent 2015 outburst in (sub)millimeter continuum toward MM1, the strongest (sub)millimeter source in the protocluster. In addition to detections toward the previously known $6.7 \mathrm{GHz}$ Class II methanol maser sites in the hot core MM2 and the UCHII region MM3 (NGC6334F), we find new maser features toward several components of MM1, along with weaker features $\sim 1^{\prime \prime}$ north, west, and southwest of MM1, and toward the non-thermal radio continuum source CM2. None of these areas have heretofore exhibited Class II methanol maser emission in three decades of observations. The strongest MM1 masers trace a dust cavity, while no masers are seen toward the strongest dust sources MM1A, 1B and 1D. The locations of the masers are consistent with a combination of increased radiative pumping due to elevated dust grain temperature following the outburst, the presence of infrared photon propagation cavities, and the presence of high methanol column densities as indicated by ALMA images of thermal transitions. The non-thermal radio emission source CM2 (2" north of MM1) also exhibits new maser emission from the excited 6.035 and $6.030 \mathrm{GHz} \mathrm{OH}$ lines. Using the Zeeman effect, we measure a line-of-sight magnetic field of +0.5 to $+3.7 \mathrm{mG}$ toward CM2. In agreement with previous studies, we also detect numerous methanol and excited $\mathrm{OH}$ maser spots toward the UCHII region MM3, with predominantly negative line-of-sight magnetic field strengths of -2 to $-5 \mathrm{mG}$ and an intriguing south-north field reversal.
\end{abstract}

Subject headings: stars: formation — masers - stars: protostars — ISM: individual objects (NGC6334I) — radio continuum: ISM — ISM: magnetic fields

\section{INTRODUCTION}

Episodic accretion in protostars is increasingly recognized as being an essential phenomenon in star formation (Kenyon et al. 1990; Evans et al. 2009). The total luminosity of a protostar scales with the instantaneous accretion rate, so variations in that rate will lead to observable brightness changes (e.g., Offner \& McKee 2011). The classical manifestations of these changes in lowmass protostars are the FU Orionis stars (FUors, Herbig 1977), which exhibit optical flares of 5 or more magnitudes followed by a slow decay, and the EX Lupi stars (EXors, Herbig 1989), which exhibit smaller, 2-3 mag-

thunter@nrao.edu

${ }^{1}$ NRAO, 520 Edgemont Rd, Charlottesville, VA 22903, USA

2 Hartebeesthoek Radio Astronomy Observatory, PO Box 443, Krugersdorp 1740, South Africa

3 SUPA, School of Physics and Astronomy, University of St. Andrews, North Haugh, St. Andrews KY16 9SS, UK

${ }^{4}$ Department of Physics and Astronomy, University of Nigeria, Carver Building, 1 University Road, Nsukka, Nigeria

5 SKA South Africa, 3rd Floor, The Park, Park Road, Pinelands, Cape Town, 7405, South Africa

6 Centre for Space Research, Physics Department, North-West University, Potchefstroom, 2520, South Africa

7 Dunlap Institute for Astronomy \& Astrophysics, University of Toronto, 50 St. George St., Toronto, Ontario, Canada, M5S $3 \mathrm{H} 4$

8 National Astronomical Observatory of Japan, Osawa 2-21-1, Mitaka, Tokyo 181-8588, Japan

${ }^{9}$ Dept of Mathematical Sciences, UNISA, PO Box 392, UNISA, 0003, South Africa

10 NRAO, PO Box O, Socorro, NM 87801, USA

11 Department of Astronomy, University of Virginia, P.O. Box 3818, Charlottesville, VA 22903, USA nitude flares but in many cases have been seen to repeat. In recent years, near-infrared surveys such as the Vista Variables in the Via Lactea (VVV, Minniti et al. 2010) and the United Kingdom Infrared Deep Sky Survey (UKIDSS, Lucas et al. 2008) have identified hundreds of high-amplitude variables, most of which have been shown to be protostars in earlier evolutionary states (Class I and II, Contreras Peña et al. 2017; Lucas et al. 2017). Outbursts in even younger and more deeplyembedded low-mass protostars have recently been detected via large increases in their mid-infrared or submillimeter emission, including a Class 0 source (HOPS383, Safron et al. 2015) from the Herschel Orion Protostar Survey (HOPS, e.g., Stutz et al. 2013, and references therein) and a Class I source (EC53, Yoo et al. 2017) from the James Clerk Maxwell Telescope (JCMT) Transient Survey (Mairs et al. 2017).

In more massive protostars, indirect evidence for episodic accretion has been inferred from outflow features seen toward high mass young stellar objects (HMYSOs). Examples include interferometric millimeter CO images of massive bipolar outflows that show high velocity bullets (e.g. Qiu \& Zhang 2009), and nearinfrared CO spectra of HMYSOs that show multiple blue-shifted absorption features analogous to the absorption features seen in FUOrs (e.g., Ellerbroek et al. 2011; Thi et al. 2010; Mitchell et al. 1991). Recently, it has become more directly clear that massive protostars also exhibit outbursts as evidenced by the $4000 L_{\odot}$ erratic variable V723 Carinae (Tapia et al. 2015), the infrared flare from the $20 M_{\odot}$ protostar powering S255IR- 
TABLE 1

VLA OBSERVING PARAMETERS

\begin{tabular}{|c|c|c|}
\hline Parameter & $\mathrm{C}$ band & $\mathrm{K}$ band \\
\hline Project code & $16 \mathrm{~B}-402$ & 10C-186 \\
\hline Observation date(s) & 2016 Oct 29,2016 Nov 19 & 2011 May 23 \\
\hline Mean epoch & 2016.9 & 2011.4 \\
\hline Configuration & A & $\mathrm{BnA}$ \\
\hline Time on source $(\min )$ & 58,58 & 92 \\
\hline Number of antennas & 26,27 & 27 \\
\hline FWHM primary beam $\left(^{\prime}\right)$ & 7 & 2 \\
\hline Baseband center frequencies $(\mathrm{GHz})$ & 5,7 & $24.059,25.406$ \\
\hline Polarization products & dual circular & dual circular \\
\hline Gain calibrator & J1717-3342 & J1717-3342 \\
\hline Bandpass calibrator & J1924-2914 & J1924-2914 \\
\hline Flux calibrator & 3C286 & 3C286 \\
\hline Spectral windows & 32 & 16 \\
\hline Digitizer resolution & 3-bit & 8-bit \\
\hline Channel spacing (narrow, wide $\mathrm{kHz}$ ) & $1.953,1000$ & 31.25 \\
\hline Total bandwidth (GHz) & 3.9 & 0.128 \\
\hline Proj. baseline range $(\mathrm{k} \lambda)$ & $4.6-980$ & $12-1030$ \\
\hline Robust clean parameter & -1.0 & +1.0 \\
\hline Cont. Resolution $\left({ }^{\prime \prime} \times{ }^{\prime \prime}\left(\right.\right.$ P.A. $\left.\left.^{\circ}\right)\right)$ & $0.63 \times 0.14(-2.9)$ & $\ldots$ \\
\hline Cont. RMS noise $\left(\mathrm{mJy} \text { beam }^{-1}\right)^{\mathrm{a}}$ & 0.022 & $\ldots$ \\
\hline Line Resolution $\left({ }^{\prime \prime} \times{ }^{\prime \prime}\left(\mathrm{P} . \mathrm{A} .^{\circ}\right)\right)$ & $0.76 \times 0.21(-3.2)^{\mathrm{b}}[6.7 \mathrm{GHz}]$ & $0.35 \times 0.26(+3.3)[24.51 \mathrm{GHz}]$ \\
\hline Line channel width $\left(\mathrm{km} \mathrm{s}^{-1}\right)$ & 0.15 & 2.0 \\
\hline RMS noise per channel (mJy beam ${ }^{-1}$ ) & $2.2^{\mathrm{c}}[6.7 \mathrm{GHz}]$ & 0.5 \\
\hline
\end{tabular}

a The continuum rms noise varies significantly with position in the image due to dynamic range limitations; the number provided here is a representative value measured near the sources.

b The $6 \mathrm{~cm}$ excited $\mathrm{OH}$ cubes have slightly larger beams due to their lower frequency: $0^{\prime \prime} .79 \times 0^{\prime \prime} \cdot 25\left(-3.2^{\circ}\right)$.

c The value reported is for Stokes I in a line-free channel, however, the spectral line sensitivity is significantly worse in channels with strong signal due to dynamic range effects. The signal-free rms noise for the RCP and LCP excited OH cubes is a factor of 1.414 times larger.

NIRS3 (Caratti o Garatti et al. 2017), and the ongoing (sub)millimeter flare of the deeply-embedded source NGC6334I-MM1 (Hunter et al. 2017). The large increases in bolometric luminosity observed in these events provide evidence for accretion outbursts similar to those predicted by hydrodynamic simulations of massive star formation (Mever et al. 2017). Identifying additional phenomena associated with these events will help to explore the mechanism of the outbursts, particularly if spectral line tracers can be identified, as they can potentially trace gas motions at high angular resolution.

Among the strongest molecular lines emitted from regions of massive star formation are a number of maser transitions (Reid \& Moran 1981; Elitzur 1992). Ever since its discovery, maser emission from protostars has exhibited eruptive phenomena, such as the three past outbursts observed in the $22 \mathrm{GHz}$ water masers in Orion KL (Abraham et al. 1981; Omodaka et al.|1999; Hirota et al. 2014), multiple events in W49N (Honma et al. 2004; Lilieström \& Gwinn $2000)$, and most recently the outburst in G25.65+1.05 (Volvach et al. 2017a, b; Lekht et al. 2017). The repeating nature of the Orion maser outbursts (Tolmachev 2011), as well as the periodic features seen toward many HMYSOs in the $6.7 \mathrm{GHz}$ methanol maser line (e.g., Goedhart et al. 2004), strongly suggest that variations in the underlying protostar could be responsible for the changes in maser emission. Such a link was suggested by Fuijisawa et al. (2012) to explain the methanol maser flare in the HMYSO G33.64-0.21. While Kumar et al. (2016) noted a similarity between the infrared light curves of several low-mass protostars in the VVV survey and the periodic methanol maser light curves of HMYSOs, these phenomena have never been observed in the same object. However, the recent methanol maser flare in S255IR (Fujisawa et al. 2015) associated with the infrared outburst in S255IR-NIRS3 has provided the first direct link between protostellar accretion outbursts and maser flares (Moscadelli et al. 2017).

Beginning in early 2015, a strong flaring of many species of masers toward the millimeter protocluster NGC6334I (Brogan et al. 2016; Hunter et al. 2006) was discovered via a regular program of single-dish maser monitoring at the Hartebeesthoek Radio Observatory (MacLeod et al. 2018). Of the four major millimeter sources in this deeply-embedded protocluster, only the UCHII region MM3 (also known as NGC6334F, Rodriguez et al. 1982) is detected in the near- and mid-infrared (e.g., Willis et al. 2013; Walsh et al. 1999; Tapia et al. 1996), despite observations at wavelengths as long as $18 \mu \mathrm{m}$ (De Buizer et al. 2002; Persi et al. 1998). While this protocluster is associated with a high-velocity, 0.5 pc-scale NE/SW bipolar outflow (Zhang et al. 2014; Qiu et al. 2011; Beuther et al. 2008; Leurini et al. 2006; Ridge \& Moore 2001; McCutcheon et al. 2000), it remains uncertain which protostar is the driving source; a component within MM1 seems the most likely, especially considering the presence of water masers there (Brogan et al. 2016). The fact that the velocity range of the flaring masers encompassed the LSR velocity of the hot molecular core thermal gas $\left(-7.2 \mathrm{~km} \mathrm{~s}^{-1}\right.$, McGuire et al. 2017; Zernickel et al. 2012; Beuther et al. 2007) provided strong evidence for an association between the flaring masers and the (sub)millimeter continuum outburst in the MM1 hot core (Hunter et al. 2017). However, the angular resolution of the maser observations was insufficient to be certain.

In this paper, we present follow-up observations of 
NGC6334I with the National Science Foundation's Karl G. Jansky Very Large Array (VLA) in $5 \mathrm{~cm}$ continuum, the $6.7 \mathrm{GHz} \mathrm{CH}_{3} \mathrm{OH}$ transition, and multiple transitions of excited-state $\mathrm{OH}$. The relatively nearby distance to the source $(1.3 \pm 0.1 \mathrm{kpc}$, inferred from the weighted mean of the two maser parallax measurements of the neighboring source I(N), Chibueze et al. 2014; Reid et al. 2014) allows us to achieve a linear resolution of $\approx 500 \mathrm{au}$. We confirm that MM1 now exhibits strong $6.7 \mathrm{GHz} \mathrm{CH}_{3} \mathrm{OH}$ maser emission. This is the first such detection of a Class II $\mathrm{CH}_{3} \mathrm{OH}$ maser from this member of the protocluster in three decades of previous interferometric observations. We also find a new source of excited $\mathrm{OH}$ maser emission toward the non-thermal radio source CM2 to the north of MM1. The locations and relative strengths of the new maser spots in relation to images of thermally excited methanol offer a unique test of maser pumping theory and provide important clues to the origin of maser emission and its response to protostellar outbursts.

\section{OBSERVATIONS}

\section{1. $V L A$}

The VLA observing parameters in the two frequency bands $(\mathrm{C}$ and $\mathrm{K})$ are presented in Table 1. In addition to the coarse resolution spectral windows used to image the continuum emission in C-band, we observed several maser transitions with high spectral resolution $2 \mathrm{MHz}$-wide correlator windows using a correlator recirculation factor of 8 . The $6.66852 \mathrm{GHz}$ (hereafter $6.7 \mathrm{GHz}$ ) transition of $\mathrm{CH}_{3} \mathrm{OH} 5(1)-6(0) \mathrm{A}^{+}$

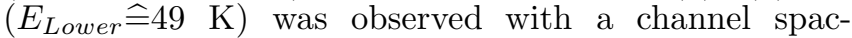
ing of $1.953 \mathrm{kHz}\left(0.0878 \mathrm{~km} \mathrm{~s}^{-1}\right)$ over a span of $90 \mathrm{~km} \mathrm{~s}^{-1}$ centered on the local standard of rest (LSR) velocity of $-7 \mathrm{~km} \mathrm{~s}^{-1}$. Three lines of excited-state $\mathrm{OH}$ maser emission were also observed: $J=1 / 2-1 / 2$, $F=0-1$ at $4.66024 \mathrm{GHz}\left(E_{\text {Lower }} \widehat{=} 182 \mathrm{~K}\right)$ observed with $0.0628 \mathrm{~km} \mathrm{~s}^{-1}$ channels over a span of $64 \mathrm{~km} \mathrm{~s}^{-1}$, and $J=5 / 2-5 / 2, F=2-2$ at $6.03075 \mathrm{GHz}$ and $F=3-3$ at

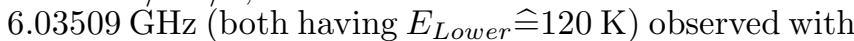
$0.0970 \mathrm{~km} \mathrm{~s}^{-1}$ channels over a span of $99 \mathrm{~km} \mathrm{~s}^{-1}$. The $6 \mathrm{GHz}$ lines were observed using the 8-bit digitizers. The data were calibrated using the VLA pipelin 12 with some additional flagging applied (the pipeline task that attempts to flag radio frequency interference was not applied to the science target in order to avoid flagging the maser features). The pipeline applies Hanning smoothing to the data, which reduces ringing from strong spectral features. The bright $6.7 \mathrm{GHz}$ maser emission was used to iteratively self-calibrate the $6.7 \mathrm{GHz}$ line emission and the resulting solutions were applied to the other line and continuum data. After excising line emission from the broad band continuum spectral windows, multiterm multi-scale clean was used with scales of 0,5 , and 15 times the image pixel size to produce the continuum image. The bright UCHII region $(\mathrm{MM} 3=\mathrm{NGC} 6334 \mathrm{~F})$ limits the dynamic range of the continuum image causing the rms noise in the image to vary significantly as a function of position. After subtracting the continuum in the uv-plane, spectral cubes of the maser emission were made with a channel spacing (and effective spectral resolution) of $0.15 \mathrm{~km} \mathrm{~s}^{-1}$. Stokes I cubes were made for the relatively unpolarized $6.7 \mathrm{GHz}$ transition, while right and left circular polarization (RCP and LCP) cubes were made for the strongly polarized excited $\mathrm{OH}$ transitions. The typical noise in a signal-free channel is $2.2 \mathrm{mJy}^{\text {beam }}{ }^{-1}$ for the $\mathrm{CH}_{3} \mathrm{OH}$ Stokes I cube, and $3.1 \mathrm{mJy}^{\mathrm{beam}}{ }^{-1}$ for the RCP and LCP $6 \mathrm{~cm}$ excited OH cubes. For all maser lines, although recirculation was used, the velocity extent of the emission is much smaller than the total bandwidth of the spectral window employed, thus none of the maser channels are affected by any spectral artifacts at high dynamic range due to phase serialization in the correlator (Sault \& Sowinski 2013).

The epoch 2011.6 K-band $(1.3 \mathrm{~cm})$ observations reported here employed narrow spectral windows (with limited total bandwidth) to target a range of spectral lines at high spectral resolution, including the $\mathrm{H} 64 \alpha$ radio recombination line (RRL) at $24.50990 \mathrm{GHz}$ that we discuss in this work. These data were calibrated in CASA using manually generated scripts prior to the development of the pipeline. The H64 $\alpha$ cube was created with a velocity resolution of $2.0 \mathrm{~km} \mathrm{~s}^{-1}$. We estimate that the absolute position uncertainties for all of the the VLA data reported here are smaller than 50 mas.

\section{2. $A L M A$}

We also present spectral line data from ALMA project 2015.A.00022.T, for which the observing parameters are described in more detail in Hunter et al. (2017). The thermal transition of methanol 11(2)-10(3) $A^{-}$at

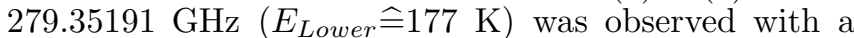
channel spacing of $976.5625 \mathrm{kHz}\left(1.05 \mathrm{~km} \mathrm{~s}^{-1}\right)$ and a velocity resolution of $1.21 \mathrm{~km} \mathrm{~s}^{-1}$. The data cube was cleaned with a robust parameter of 0 and has a beamsize of $0^{\prime \prime} .29 \times 0^{\prime \prime} .22$ at position angle $-84^{\circ}$. The $\mathrm{rms}$ noise achieved is 1.7 mJy beam ${ }^{-1}$ in $1.1 \mathrm{~km} \mathrm{~s}^{-1}$ wide channels. We constructed a moment image of the peak intensity across the 22 channels spanning the entire line. We estimate that the absolute position uncertainties for the ALMA data presented here are smaller than 50 mas.

\section{RESULTS}

\subsection{Radio continuum}

Contours from the new $5 \mathrm{~cm}$ continuum image are shown in Fig. 1 alongside those from the epoch 2011.4 image (Brogan et al. 2016). The morphology and intensity of the emission are consistent given the difference in synthesized beams, and the lower sensitivity and image fidelity of the earlier data. In particular, the compact non-thermal source CM2 reported by Brogan et al. (2016) has persisted to the current epoch, as has the north-south jet-like feature centered on the grouping of millimeter sources designated MM1. The fitted position of the continuum emission from the point source CM1 located outside the field of view of Fig. 1 1 to the west of the central protocluster (Brogan et al. 2016) agrees between the 2011.4 and 2016.9 epochs to within $0{ }^{\prime \prime} 03$; thus, there is no evidence for any significant instrumental shift in astrometry between the two epochs. We also note that the proper motion of the parent cloud (measured by the Very Long Baseline Array, Wu et al. 2014) accumulated

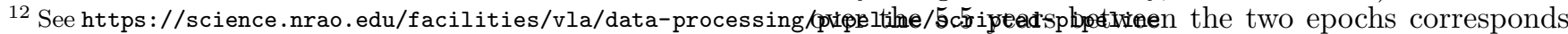
for more information. to only $(+1.7,-12)$ milliarcsec, which is undetectable 

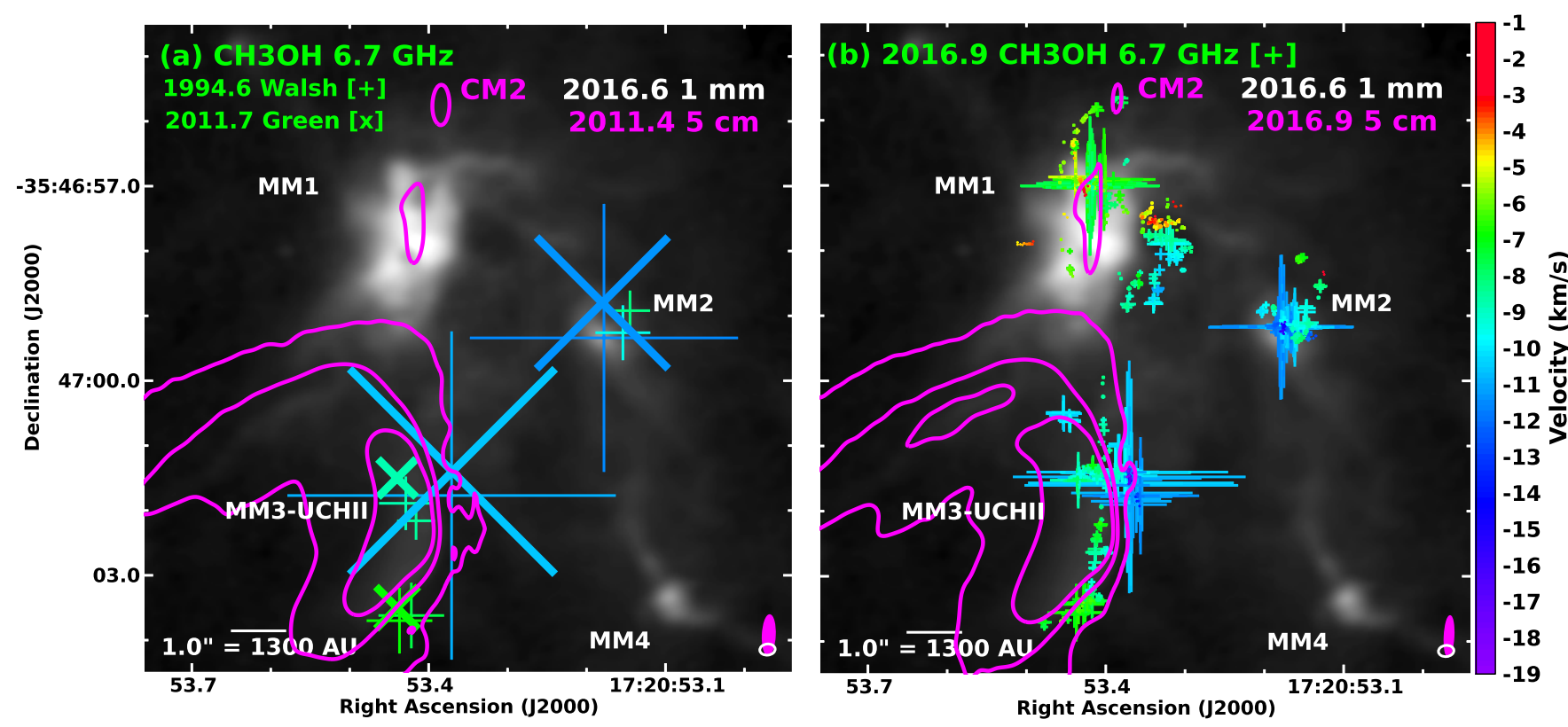

FIG. 1.- (a) The methanol maser positions observed by the Australia Telescope Compact Array (ATCA) on July 31, 1994 (Walsh et al. $1998,+$ symbols, detection threshold $\approx 1 \mathrm{Jy}$ ) and September 2,2011 (Green et al.|2015, x symbols, detection threshold $\approx 0.6 \mathrm{Jy}$ ) are overlaid on an epoch 2016.6 ALMA $1 \mathrm{~mm}$ continuum image in greyscale (Hunter et al.|2017). Epoch 2011.4 VLA 5 cm continuum contours (Brogan et al. 2016) are also overlaid in magenta at levels of $(4,260$, and 600$) \times$ the rms level of $3.7 \times 10^{-2} \mathrm{mJy} \mathrm{beam}^{-1}$. Maser intensity is indicated by the diameter of the symbol (the diameter is proportional to the square root of the intensity), while velocity is indicated by the color of the symbol. The millimeter continuum sources are labeled for reference. (b) Same as (a) but the epoch $2016.95 \mathrm{~cm}$ continuum (magenta countours) and methanol masers (+ symbols) observed with the VLA are overlaid. The continuum contours are (4, 260, and $600) \times$ the rms level of $2.2 \times 10^{-2} \mathrm{mJy}_{\text {beam }}^{-1}$. The synthesized beam shape is slightly different between the epochs.

TABLE 2

Fitted properties of the 6.7 GHz methanol masers

\begin{tabular}{cccccr}
\hline \hline Number $^{\mathrm{a}}$ & Association & $\begin{array}{c}\text { Velocity channel } \\
\left(\mathrm{km} \mathrm{s}^{-1}\right)\end{array}$ & \multicolumn{2}{c}{$\begin{array}{c}\text { Fitted Position }(\mathrm{J} 2000) \\
\text { R.A. }\end{array}$} & $\begin{array}{c}\text { Flux Density } \\
(\mathrm{Jy})^{\mathrm{b}}\end{array}$ \\
\hline 1 & UCHII-Met4 & -18.80 & $17: 20: 53.3729$ & $-35: 47: 01.474$ & $0.0119(0.0015)$ \\
2 & UCHII-Met4 & -18.65 & $17: 20: 53.3721$ & $-35: 47: 01.382$ & $0.00893(0.00148)$ \\
3 & UCHII-Met4 & -17.75 & $17: 20: 53.3703$ & $-35: 47: 01.456$ & $0.0114(0.0015)$ \\
4 & UCHII-Met4 & -17.60 & $17: 20: 53.3715$ & $-35: 47: 01.356$ & $0.0119(0.0015)$ \\
5 & UCHII-Met4 & -17.45 & $17: 20: 53.3705$ & $-35: 47: 01.393$ & $0.00988(0.00147)$ \\
6 & UCHII-Met4 & -17.30 & $17: 20: 53.3696$ & $-35: 47: 01.361$ & $0.0110(0.0015)$ \\
7 & UCHII-Met4 & -17.15 & $17: 20: 53.3663$ & $-35: 47: 01.416$ & $0.0109(0.0015)$ \\
8 & UCHII-Met4 & -17.00 & $17: 20: 53.3710$ & $-35: 47: 01.394$ & $0.0111(0.0015)$ \\
9 & UCHII-Met4 & -16.85 & $17: 20: 53.3685$ & $-35: 47: 01.503$ & $0.0150(0.0014)$ \\
10 & UCHII-Met4 & -16.70 & $17: 20: 53.3684$ & $-35: 47: 01.496$ & $0.0171(0.0015)$ \\
& & & & & \\
\hline
\end{tabular}

\footnotetext{
a Entries are sorted by velocity

b Uncertainties in the fitted value are listed in parentheses, and do not include the flux calibration uncertainty.

(This table is available in its entirety in a machine-readable form in the online journal. A portion is shown here for guidance regarding its form and content.)
}

at the current resolution. Further details about the centimeter wavelength continuum emission will be presented in a future multi-wavelength paper (Brogan et al. 2018, in preparation).

\section{2. $6.7 \mathrm{GHz}$ methanol masers}

Because the degree of circular polarization of the $6.7 \mathrm{GHz}$ maser emission is very low, we analyzed the Stokes I cube. Strong emission appears in many directions within the central protocluster. The spectrum toward any given pixel typically extends over several $\mathrm{km} \mathrm{s}^{-1}$ with components of rather different strength and width appearing at different velocities, and thus is not amenable to Gaussian spectral profile fitting. Instead, we fit each channel of the cube that had significant emission ( $>6 \sigma$, measured independently for each channel) with an appropriate number of two-dimensional Gaussian sources. Because the spots of maser emission are likely smaller than our synthesized beam (Table 1), we fixed the fitted shape parameters to the synthesized beam. We used the pixel position of each emission peak as the initial guess for the centroid position of each fit. In channels with complicated emission, we also set the intensity of each peak as an initial guess. After manual inspection of all the fits, a few of the weakest features that formally met the sensitivity criterion could be at- 

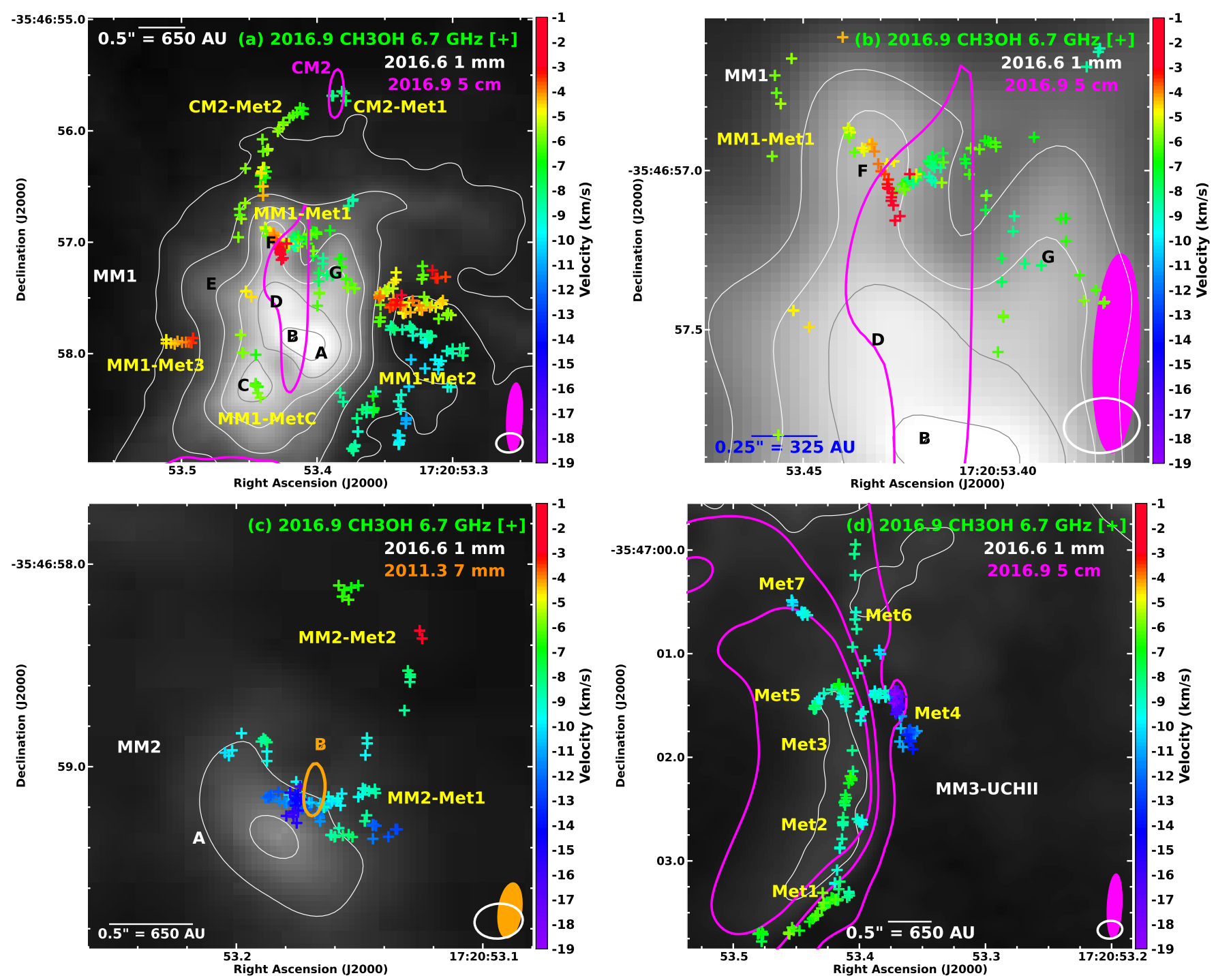

Fig. 2.- Panels (a), (b), (c), and (d) show close up views of regions with $6.7 \mathrm{GHz} \mathrm{CH}_{3} \mathrm{OH}$ masers from Fig. 10; the magenta VLA $5 \mathrm{~cm}$ contour levels are the same. The greyscale and white (or gray) contours show the ALMA $1 \mathrm{~mm}$ dust emission, with contour levels of (a) 15, 56, 140, 245, 364, $490 \mathrm{mJy}_{\text {beam }}{ }^{-1}$; (b) 140, 245, 364, $490 \mathrm{mJy}_{\text {beam }}^{-1}$, (c) 50, $140 \mathrm{mJy} \mathrm{beam}^{-1}$; and (d) $21 \mathrm{mJy} \mathrm{beam}^{-1}$. Panel (c) shows a single $7 \mathrm{~mm}$ contour (in orange) denoting the intensity level $0.72 \mathrm{mJy}$ beam $^{-1}$ for the epoch 2011.3 to indicate the position of MM2B (Brogan et al. 2016). In panels (a), (b), and (c) the black, white, or orange letters denote the compact dust continuum sources defined by Brogan et al. (2016). In each panel, the maser associations from Table 3 are labelled in yellow for reference, and the synthesized beam sizes are shown in the lower right-hand corners. The maser locations are marked by + symbols with fixed size.

tributed to imaging artifacts in dynamic-range limited channels and were subsequently discarded. The fitted per-channel positions and per-channel flux densities of the masers are given in Table 2, and their positions and relative intensities are shown in Figure 1 b. For comparison, we also show previously published maser spots from two representative studies in Figure 19. As this figure demonstrates, the general location and strength of the masers toward MM2 and MM3 are consistent with past observations (Walsh et al. 1998; Green et al. 2015). To summarize the salient maser properties in a compact format we have identified several distinct maser "associations" toward each of the continuum sources with detected $6.7 \mathrm{GHz}$ maser emission: MM1, MM2, CM2, and the UCHII region MM3. The assignment of each fitted spot to an association is provided in a column in Table 2, and the properties and general locations of the associa- tions are summarized in Table 3 and Figure 2a, b, c, and d, respectively.

Lower limits to the peak $6.7 \mathrm{GHz}$ maser brightness temperatures $\left(T_{B}\right)$ for the associations are also given in Table 3 derived using the peak intensity and the synthesized beam size (Table 11). Even given our modest resolution, lower limits as high as $T_{B}=8.5 \times 10^{5}$, $1.1 \times 10^{8}, 1.5 \times 10^{8}$, and $3.1 \times 10^{8} \mathrm{~K}$ are found for the CM2, MM1, MM2, and MM3-UCHII regions, respectively. A number of studies using very long baseline interferometry (VLBI) have explored the intrinsic size of $6.7 \mathrm{GHz}$ masers, with several finding that significant flux (up to $50 \%$ ) can be missed by very high angular resolution observations, suggesting a core-halo type of morphology with a very compact core of a few au and extended halos of a few hundred au (i.e., of order the minor axis of our synthesized beam, 0 "'2) (see for example Minier et al. 
TABLE 3

Summary OF 6.7 GHz METHANOL MASER ASSOCIATIONS

\begin{tabular}{|c|c|c|c|c|c|c|}
\hline Association & $\begin{array}{l}\text { Centroid pos } \\
\text { R.A. }\end{array}$ & $\begin{array}{l}\text { ition }(\mathrm{J} 2000) \\
\text { Dec. }\end{array}$ & $\begin{array}{l}\text { Integrated flux } \\
\left(\mathrm{Jy} \mathrm{km} \mathrm{s}^{-1}\right)\end{array}$ & $\begin{array}{l}\text { Velocity min, peak, max } \\
\qquad\left(\mathrm{km} \mathrm{s}^{-1}\right)\end{array}$ & $\begin{array}{l}\text { Peak flux density } \\
\text { (Jy) }\end{array}$ & $\begin{array}{c}T_{B}{ }^{\mathrm{a}} \\
\left(10^{6} \mathrm{~K}\right)\end{array}$ \\
\hline CM2-Met1 & $17: 20: 53.381$ & $-35: 46: 55.68$ & 2.00 & $-8.00,-7.55,-7.40$ & 5.03 & 0.849 \\
\hline CM2-Met2 & $17: 20: 53.413$ & $-35: 46: 55.84$ & 2.72 & $-6.80,-6.65,-5.30$ & 4.98 & 0.840 \\
\hline CM2 Total & & & 4.72 & $-8.00,-7.55,-5.30$ & & \\
\hline MM1-Met1 & $17: 20: 53.417$ & $-35: 46: 56.98$ & 741 & $-8.60,-7.25,-1.55$ & 545 & 92.0 \\
\hline MM1-Met2 & $17: 20: 53.327$ & $-35: 46: 58.01$ & 70.8 & $-11.00,-9.35,-2.75$ & 55.4 & 9.35 \\
\hline MM1-Met3 & $17: 20: 53.499$ & $-35: 46: 57.90$ & 0.0925 & $-4.55,-3.50,-3.20$ & 0.111 & 0.0188 \\
\hline MM1-MetC & $17: 20: 53.439$ & $-35: 46: 58.30$ & 0.924 & $-8.30,-6.20,-5.60$ & 1.51 & 0.255 \\
\hline MM1 Total & & & 813 & $-11.00,-7.25,-1.55$ & & \\
\hline MM2-Met1 & $17: 20: 53.174$ & $-35: 46: 59.17$ & 644 & $-15.95,-11.15,-7.70$ & 583 & 98.4 \\
\hline MM2-Met2 & $17: 20: 53.139$ & $-35: 46: 58.39$ & 3.55 & $-8.30,-7.85,-2.30$ & 5.59 & 0.943 \\
\hline MM2 Total & & & 648 & $-15.95,-11.15,-2.30$ & & \\
\hline UCHII-Met1 & $17: 20: 53.430$ & $-35: 47: 03.46$ & 87.7 & $-9.35,-6.65,-5.60$ & 93.0 & 15.7 \\
\hline UCHII-Met2 & $17: 20: 53.410$ & $-35: 47: 02.56$ & 13.7 & $-9.35,-8.00,-6.35$ & 10.9 & 1.83 \\
\hline UCHII-Met3 & $17: 20: 53.405$ & $-35: 47: 02.04$ & 0.203 & $-8.15,-8.00,-8.00$ & 0.707 & 0.119 \\
\hline UCHII-Met4 & $17: 20: 53.370$ & $-35: 47: 01.52$ & 1760 & $-18.80,-10.40,-8.15$ & 1820 & 307 \\
\hline UCHII-Met5 & $17: 20: 53.430$ & $-35: 47: 01.49$ & 61.2 & $-9.50,-8.60,-6.80$ & 88.9 & 15.0 \\
\hline UCHII-Met6 & $17: 20: 53.389$ & $-35: 47: 00.91$ & 5.80 & $-10.10,-10.10,-8.00$ & 16.9 & 2.86 \\
\hline UCHII-Met7 & $17: 20: 53.451$ & $-35: 47: 00.53$ & 23.2 & $-10.25,-10.10,-9.05$ & 36.3 & 6.13 \\
\hline UCHII Total & $\ldots$ & $\ldots$ & 1950 & $-18.80,-10.40,-5.60$ & $\ldots$ & $\ldots$ \\
\hline Grand Total & $\ldots$ & $\ldots$ & 3420 & $-18.80,-10.40,-1.55$ & $\ldots$ & $\ldots$ \\
\hline
\end{tabular}

${ }^{a}$ Lower limits to the brightness temperature $T_{B}$ using the synthesized beam; the physical sizes of the maser spots are likely to be smaller than the beam $(\sim 520 \mathrm{au})$.

2002; Harvey-Smith \& Cohen 2006). Therefore, we are unlikely to be missing significant flux, but it is possible that the maser spot cores have significantly higher $T_{B}$ than our current lower limits.

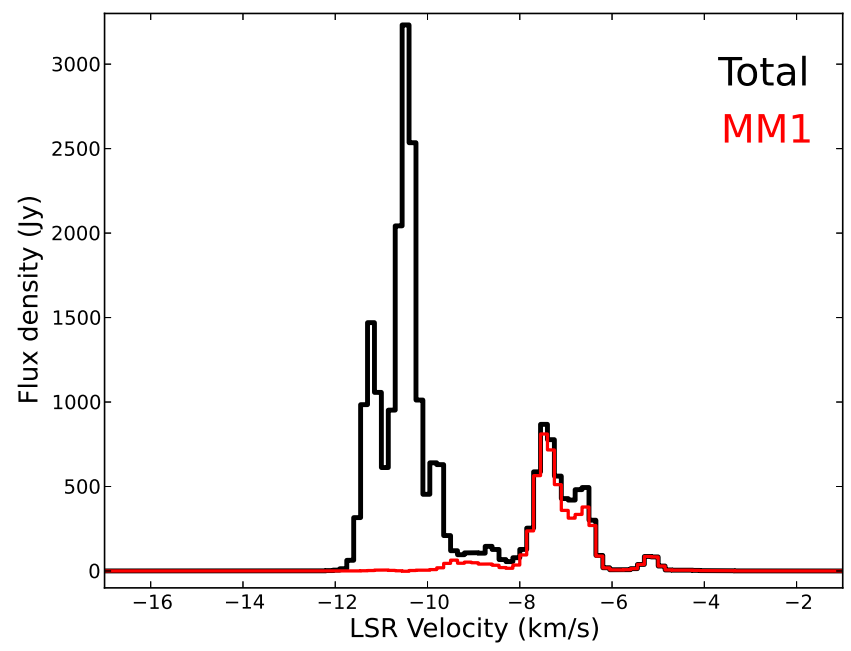

FIG. 3.- Integrated Stokes I spectra from the epoch 2016.9 6.7 $\mathrm{GHz}$ methanol maser cube integrated over all the maser features (black spectrum) and over an elliptical region surrounding only the maser features associated with MM1 (red spectrum).

\subsubsection{MM1 and CM2 region}

In epoch 2016.9, many $6.7 \mathrm{GHz} \mathrm{CH}_{3} \mathrm{OH}$ maser features have appeared within the dust continuum source MM1 (including the associations MM1-Met1 and MM1MetC) and along its western periphery (MM1-Met2), eastern periphery (MM1-Met3), and northern peripheries (CM2-Met1, and CM2-Met2). A zoom to the area around MM1 and CM2 is shown in Figure 2a. These are the first detections of Class II methanol maser emission toward this region within the NGC6334I protocluster from nearly 30 years of interferometric observations, all of which were sufficiently sensitive to detect masers of this strength. Previous epochs at 6.7 GHz include January 1992 (Norris et al. 1993), May 1992 (Ellingsen 1996; Ellingsen et al. 1996), July 1994 (Walsh et al. 1998), July 1995 (Caswell 1997), September 1999 (Dodson \& Moriartv 2012), March 2005 (Krishnan et al. 2013), May 2011 (Brogan et al. 2016), and August 2011 (Green et al. 2015). In all of these epochs, detections of this maser transition in this field originate from the UCHII region (NGC6334F) and MM2. In addition, the original VLBI imaging of the Class II 12.2 $\mathrm{GHz}$ methanol maser transition did not detect any features toward MM1 (Norris et al.|1988), nor did the May 1992 ATCA observations (Ellingsen et al. 1996).

The brightest $6.7 \mathrm{GHz}$ features in MM1 (peak = 545 Jy at $-7.25 \mathrm{~km} \mathrm{~s}^{-1}$ ) reside in the valley of dust continuum emission between the $1 \mathrm{~mm}$ dust continuum sources MM1F and MM1G (in the MM1-Met1 association). This area lies about 1000 au north of the hypercompact HII region MM1B, which is the proposed central driving source of the millimeter outburst (Hunter et al. 2017). The velocity of the brightest feature coincides with the LSR velocity of the thermal molecular gas $\left(-7.3 \mathrm{~km} \mathrm{~s}^{-1}\right.$, Zernickel et al. 2012). The integrated spectrum of MM1 (all associations shown in Fig. 2a) compared to the total field (Figure 3) further demonstrates that the dominant emission from this region arises from -6 to $-8 \mathrm{~km} \mathrm{~s}^{-1}$.

Within the MM1-Met1 association (see Fig. 2b), a line of strong spots form the western side of a "V"-shaped structure, with a weaker line of redshifted features south of MM1F forming the eastern side. Additional strong features appear on the dust continuum source MM1F while weaker features near the LSR velocity lie around MM1G and extend northward from MM1F (including the association CM2-Met2). The northernmost spots (in association CM2-Met1) are coincident with the non- 
thermal radio source CM2. Another set of near-LSR features (MM1-MetC) coincide with the millimeter source MM1C, however the three brightest and most central millimeter sources (MM1A, B, and D) are notably lacking in any maser emission. A large number of moderate strength, primarily blueshifted masers (MM1-Met2) lie in cavities of the dust emission located west and southwest of MM1A. Finally, a number of weak, moderately redshifted features (MM1-Met3) lie eastward in an depression in the dust emission that originates between MM1C and MM1E.

\subsubsection{MM2 region}

A zoom to the area around MM2 is shown in Figure 2r. The strongest masers (MM2-Met1 in Tables 22 and 3) are situated between the $1 \mathrm{~mm}$ source MM2A and the $7 \mathrm{~mm}$ source MM2B and are predominantly blueshifted by 5$10 \mathrm{~km} \mathrm{~s}^{-1}$ from the LSR velocity of the thermal molecular gas. A subgroup of masers near the LSR velocity are centered just west of the MM2B. Weaker masers lie $\sim 1^{\prime \prime}$ to the northwest (MM2-Met2 in Tables 2 and 3) including two weak redshifted spots.

\subsubsection{MM3 region (UCHII region)}

A zoom to the area around MM3 is shown in Figure $2 \mathrm{~d}$. The arrangement of $6.7 \mathrm{GHz}$ maser spots toward the UCHII region is strikingly filamentary, and seems to closely trace the shape of the $5 \mathrm{~cm}$ continuum emission, especially toward the southern and middle regions. These masers may arise in a layer of compressed gas just outside the UCHII region. Most of these spots lie near the LSR velocity, becoming somewhat blueshifted in the northern half. The strongest spots (Met4) are significantly blueshifted (by up to $12 \mathrm{~km} \mathrm{~s}^{-1}$ ) and cluster around a small knot in the continuum emission located about $1^{\prime \prime}$ northwest of the $5 \mathrm{~cm}$ UCHII region peak.

\subsection{Excited-state $\mathrm{OH} 6.030$ and $6.035 \mathrm{GHz}$ transitions}

NGC6334I is one of the strongest known sources of the $5 \mathrm{~cm}$ excited-state OH masers (Caswell \& Vaile 1995; Zuckerman et al. 1972; Gardner et al. 1970). Although the excited $\mathrm{OH}$ spectra are generally simpler than those of the $6.7 \mathrm{GHz}$ methanol, in order to match the methanol maser fitting procedure, we also fit the excited $\mathrm{OH}$ maser properties using the channel by channel approach. Because the emission in these $\mathrm{OH}$ transitions is strongly circularly polarized, we fit the image cubes of RCP and LCP emission independently. The fitted positions and flux densities of the two transitions $(6.030$ and $6.035 \mathrm{GHz})$ are given in Tables 4, 5, 6 and 7. The maser spots are shown in Figure 4. Note we have not attempted to correct the flux densities for beam squint, but this effect will be small $(\sim 1.2 \%)$ toward the inner parts of the $7^{\prime}$ primary beam where the masers are located (beam squint does not affect the Zeeman frequency splitting). Since all of the excited $\mathrm{OH}$ masers are found in the same general areas as the $6.7 \mathrm{GHz}$ methanol detections, the labels for the $\mathrm{OH}$ associations have the same numerical index for a given region, for example UCHII-Met1 has an analogous excited $\mathrm{OH}$ maser association UCHII-OH1. The spatial distribution of the two $\mathrm{OH}$ transitions is similar, with $6.035 \mathrm{GHz}$ being significantly stronger in most locations and showing additional associations ( $\mathrm{OH} 5$ and $\mathrm{OH} 7)$ toward the northern portion of the UCHII region (Figure 51). The positions of the seven associations UCHII-OH1 through UCHII-OH7 are closely matched to those of the $6.7 \mathrm{GHz}$ maser associations UCHII-Met1 through UCHII-Met7.

Integrated spectra of the cubes are shown in Figure 6 . These are similar to previous single-dish spectra toward this region (Caswell 2003; Avison et al. 2016), with the exception of the strong new association toward CM2, which now dominates the emission near the LSR velocity, peaking at $-7.7 \mathrm{~km} \mathrm{~s}^{-1}$. Both lines were also previously observed interferometrically (Knowles et al. 1973; Caswell 1997; Green et al. 2015), with the highest resolution images produced with the Long Baseline Array (LBA) in January 2001 (Caswell et al. 2011), which showed the ridge of spots along the UCHII region, but no feature toward CM2 has ever been reported. We thus conclude that the CM2 feature is associated with the millimeter outburst. It is notable that the flux ratio of the $6.035 / 6.030$ lines toward CM2 is abnormally low $(1.2-1.8)$ compared to the rest of the spots, which have a median ratio of 23, similar to the historical ratio of 25 noted by Knowles et al. (1976). At the current resolution, a lower limit to the peak brightness temperature of the 6.030 and $6.035 \mathrm{GHz}$ masers is $3 \times 10^{6} \mathrm{~K}$ and $5 \times 10^{7} \mathrm{~K}$, respectively (UCHII-OH4-1b, see §3.3.1). From LBA observations toward NGC6334I, Caswell et al. (2011) found the excited $\mathrm{OH}$ spot sizes to be smaller than 20 mas for the UCHII region masers, and intensities within a factor of 2 of those reported here. From much higher angular resolution European VLBI Network (EVN) observations of the same excited $\mathrm{OH}$ transitions in W3(OH), Fish \& Siouwerman (2007) find typical maser spot sizes of order 5 mas or smaller, which would translate to 7.5 mas or smaller at the nearer distance of NGC6334I. Thus, the true $\mathrm{OH}$ brightness temperatures are likely to be orders of magnitude higher than reported here.

\subsubsection{Zeeman splitting}

The identification of Zeeman pairs can be challenging with an angular resolution of $0^{\prime \prime} .79 \times 0^{\prime \prime} \cdot 25(\sim 520 \mathrm{au})$, i.e. significantly larger than the likely physical size of a single maser "spot". We have nevertheless used the following procedure to kinematically and spatially separate (within the limitations of the data) the individual masing regions. For both transitions, we first identified maser "spots" of contiguous velocity components with fitted positions that were within $1 / 3$ of the minor axis of the synthesized beam, independently for RCP and LCP. These independent RCP and LCP spot positions were then compared with each other and deemed to be a Zeeman pair if their intensity weighted centroid positions were within $1 / 3$ of the minor axis of the synthesized beam. Then the RCP and LCP profiles for each Zeeman pair were independently fitted using Gaussian profiles. In a few cases, spatially coincident Zeeman pairs consisted of more than one velocity component and these were further separated into independent "spots", though the spectral Gaussian fits per polarization were derived simultaneously. The derived parameters, including the fitted flux density and line center velocity $\left(V_{\text {center }}\right)$ for the Zeeman pairs for both transitions are given in Table 8 . The spots have been named according to their association, and then an additional number in increasing velocity order; spots with multiple velocity components (at 
TABLE 4

Fitted properties of the OH 6.030 GHz Masers (LEFt CirCular POlarization)

\begin{tabular}{|c|c|c|c|c|c|}
\hline \multirow[t]{2}{*}{ Number ${ }^{a}$} & \multirow[t]{2}{*}{ Association } & \multirow{2}{*}{$\begin{array}{l}\text { Velocity channel } \\
\qquad\left(\mathrm{km} \mathrm{s}^{-1}\right)\end{array}$} & \multicolumn{2}{|c|}{ Fitted Position (J2000) } & \multirow{2}{*}{$\begin{array}{l}\text { Flux Density } \\
(\mathrm{Jy})^{\mathrm{b}}\end{array}$} \\
\hline & & & R.A. & Dec. & \\
\hline 1 & UCHII-OH4 & -11.00 & $17: 20: 53.3718$ & $-35: 47: 01.573$ & $0.0241(0.0025)$ \\
\hline 2 & UCHII-OH4 & -10.85 & $17: 20: 53.3722$ & $-35: 47: 01.573$ & $0.0903(0.0022)$ \\
\hline 3 & UCHII-OH6 & -10.85 & $17: 20: 53.3975$ & $-35: 47: 00.737$ & $0.0294(0.0022)$ \\
\hline 4 & UCHII-OH4 & -10.70 & $17: 20: 53.3712$ & $-35: 47: 01.531$ & $0.326(0.002)$ \\
\hline 5 & UCHII-OH6 & -10.70 & $17: 20: 53.3967$ & $-35: 47: 00.768$ & $0.203(0.002)$ \\
\hline 6 & UCHII-OH4 & -10.55 & $17: 20: 53.3709$ & $-35: 47: 01.525$ & $2.84(0.01)$ \\
\hline 7 & UCHII-OH6 & -10.55 & $17: 20: 53.3972$ & $-35: 47: 00.775$ & $0.348(0.004)$ \\
\hline 8 & UCHII-OH4 & -10.40 & $17: 20: 53.3709$ & $-35: 47: 01.525$ & $13.3(0.1)$ \\
\hline 9 & UCHII-OH6 & -10.40 & $17: 20: 53.3979$ & $-35: 47: 00.780$ & $0.253(0.007)$ \\
\hline 10 & UCHII-OH4 & -10.25 & $17: 20: 53.3709$ & $-35: 47: 01.524$ & $16.6(0.1)$ \\
\hline
\end{tabular}

a Entries are sorted by velocity

b Uncertainties in the fitted value are listed in parentheses, and do not include the flux calibration uncertainty.

(This table is available in its entirety in a machine-readable form in the online journal. A portion is shown here for guidance regarding its form and content.)

TABLE 5

FitTED PROPERTIES OF THE OH 6.030 GHz MASERS (RIGHT CIRCUlAR POlarizATION)

\begin{tabular}{|c|c|c|c|c|c|}
\hline \multirow[t]{2}{*}{ Number $^{\mathrm{a}}$} & \multirow[t]{2}{*}{ Association } & \multirow{2}{*}{$\begin{array}{l}\text { Velocity channel } \\
\quad\left(\mathrm{km} \mathrm{s}^{-1}\right)\end{array}$} & \multicolumn{2}{|c|}{ Fitted Position (J2000) } & \multirow{2}{*}{$\begin{array}{l}\text { Flux Density } \\
(\mathrm{Jy})^{\mathrm{b}}\end{array}$} \\
\hline & & & R.A. & Dec. & \\
\hline 1 & UCHII-OH4 & -11.30 & $17: 20: 53.3687$ & $-35: 47: 01.536$ & $0.0305(0.0021)$ \\
\hline 2 & UCHII-OH4 & & $17: 20: 53.3704$ & $-35: 47: 01.533$ & $0.113(0.002)$ \\
\hline 3 & UCHII-OH4 & -11.00 & $17: 20: 53.3709$ & $-35: 47: 01.527$ & $0.973(0.003)$ \\
\hline 4 & UCHII-OH4 & -10.85 & 17:20:53.3709 & $-35: 47: 01.525$ & $6.56(0.01)$ \\
\hline 5 & UCHII-OH4 & -10.70 & 17:20:53.3709 & $-35: 47: 01.524$ & $13.0(0.1)$ \\
\hline 6 & UCHII-OH4 & -10.55 & 17:20:53.3709 & $-35: 47: 01.523$ & $6.78(0.01)$ \\
\hline 7 & UCHII-OH4 & -10.40 & $17: 20: 53.3708$ & $-35: 47: 01.527$ & $0.639(0.003)$ \\
\hline 8 & UCHII-OH6 & -10.40 & $17: 20: 53.3964$ & $-35: 47: 00.796$ & $0.128(0.003)$ \\
\hline 9 & UCHII-OH4 & -10.25 & $17: 20: 53.3702$ & $-35: 47: 01.547$ & $0.0517(0.0027)$ \\
\hline 10 & UCHII-OH6 & -10.25 & $17: 20: 53.3967$ & $-35: 47: 00.776$ & $0.332(0.003)$ \\
\hline
\end{tabular}

a Entries are sorted by velocity

b Uncertainties in the fitted value are listed in parentheses, and do not include the flux calibration uncertainty.

(This table is available in its entirety in a machine-readable form in the online journal. A portion is shown here for guidance regarding its form and content.)

TABLE 6

Fitted properties of the OH 6.035 GHz Masers (Left CirCular POlarization)

\begin{tabular}{|c|c|c|c|c|c|}
\hline \multirow[t]{2}{*}{ Number $^{\mathrm{a}}$} & \multirow[t]{2}{*}{ Association } & \multirow{2}{*}{$\begin{array}{l}\text { Velocity channel } \\
\qquad\left(\mathrm{km} \mathrm{s}^{-1}\right)\end{array}$} & \multicolumn{2}{|c|}{ Fitted Position (J2000) } & \multirow{2}{*}{$\begin{array}{l}\text { Flux Density } \\
(\mathrm{Jy})^{\mathrm{b}}\end{array}$} \\
\hline & & & R.A. & Dec. & \\
\hline 1 & UCHII-OH4 & -12.05 & $17: 20: 53.3719$ & $-35: 47: 01.507$ & $0.0231(0.0022)$ \\
\hline 2 & UCHII-OH4 & -11.90 & $17: 20: 53.3709$ & $-35: 47: 01.555$ & $0.0281(0.0024)$ \\
\hline 3 & UCHII-OH4 & -11.75 & $17: 20: 53.3717$ & $-35: 47: 01.551$ & $0.0376(0.0024)$ \\
\hline 4 & UCHII-OH4 & -11.60 & $17: 20: 53.3718$ & $-35: 47: 01.558$ & $0.0672(0.0021)$ \\
\hline 5 & UCHII-OH4 & -11.45 & $17: 20: 53.3723$ & $-35: 47: 01.560$ & $0.0972(0.0025)$ \\
\hline 6 & UCHII-OH4 & -11.15 & $17: 20: 53.3638$ & $-35: 47: 01.863$ & $0.895(0.009)$ \\
\hline 7 & UCHII-OH4 & -11.15 & $17: 20: 53.3712$ & $-35: 47: 01.544$ & $5.35(0.01)$ \\
\hline 8 & UCHII-OH4 & -11.00 & $17: 20: 53.3631$ & $-35: 47: 01.858$ & $1.06(0.01)$ \\
\hline 9 & UCHII-OH4 & -11.00 & $17: 20: 53.3712$ & $-35: 47: 01.543$ & $30.0(0.1)$ \\
\hline 10 & UCHII-OH4 & -10.85 & $17: 20: 53.3712$ & $-35: 47: 01.542$ & $36.9(0.1)$ \\
\hline
\end{tabular}

a Entries are sorted by velocity

b Uncertainties in the fitted value are listed in parentheses, and do not include the flux calibration uncertainty.

(This table is available in its entirety in a machine-readable form in the online journal. A portion is shown here for guidance regarding its form and content.) 

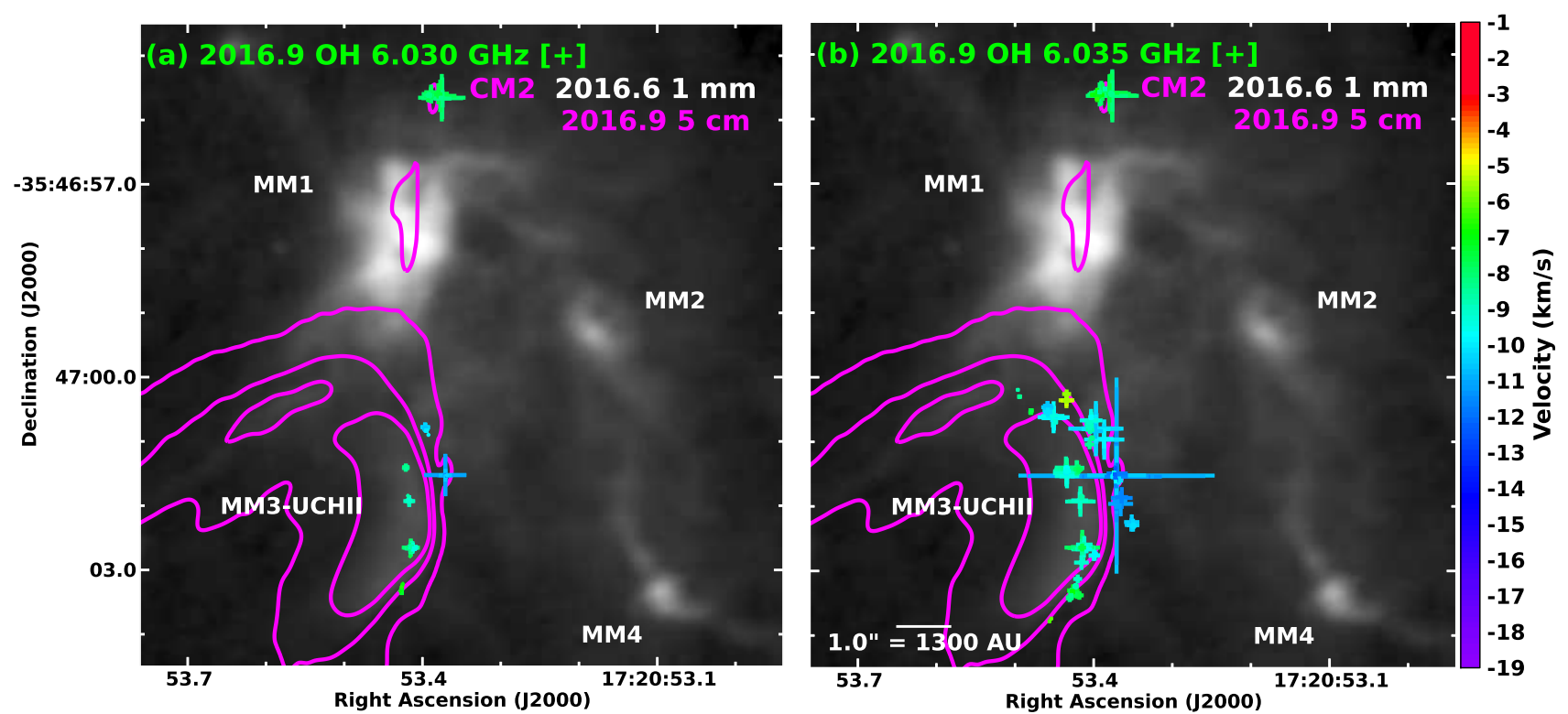

FIG. 4.- Excited-state OH masers: (a) The fitted positions of the $6.030 \mathrm{GHz}$ transition (+ symbols) are overlaid on an epoch 2016.6 ALMA $1 \mathrm{~mm}$ continuum image in greyscale (Hunter et al. 2017). In both panels, the VLA $5 \mathrm{~cm}$ continuum contours from epoch 2016.9 are overlaid in magenta with contour levels the same as Fig. 1 b. As in Fig. 1 the maser intensity is indicated by the size of the symbol, while velocity is indicated by the color. Continuum sources are labeled for reference; (b) Same as (a) but for the $6.035 \mathrm{GHz}$ transition.

TABLE 7

Fitted properties of the OH 6.035 GHz masers (Right Circular polarization)

\begin{tabular}{cccccc}
\hline \hline Number $^{\text {a }}$ & Association & $\begin{array}{c}\text { Velocity channel } \\
\left(\mathrm{km} \mathrm{s}^{-1}\right)\end{array}$ & \multicolumn{2}{c}{ Fitted Position (J2000) } \\
R.A. & Flux Density \\
$(\mathrm{Dy})^{\mathrm{b}}$
\end{tabular}

${ }^{a}$ Entries are sorted by velocity

b Uncertainties in the fitted value are listed in parentheses, and do not include the flux calibration uncertainty.

(This table is available in its entirety in a machine-readable form in the online journal. A portion is shown here for guidance regarding its form and content.) 


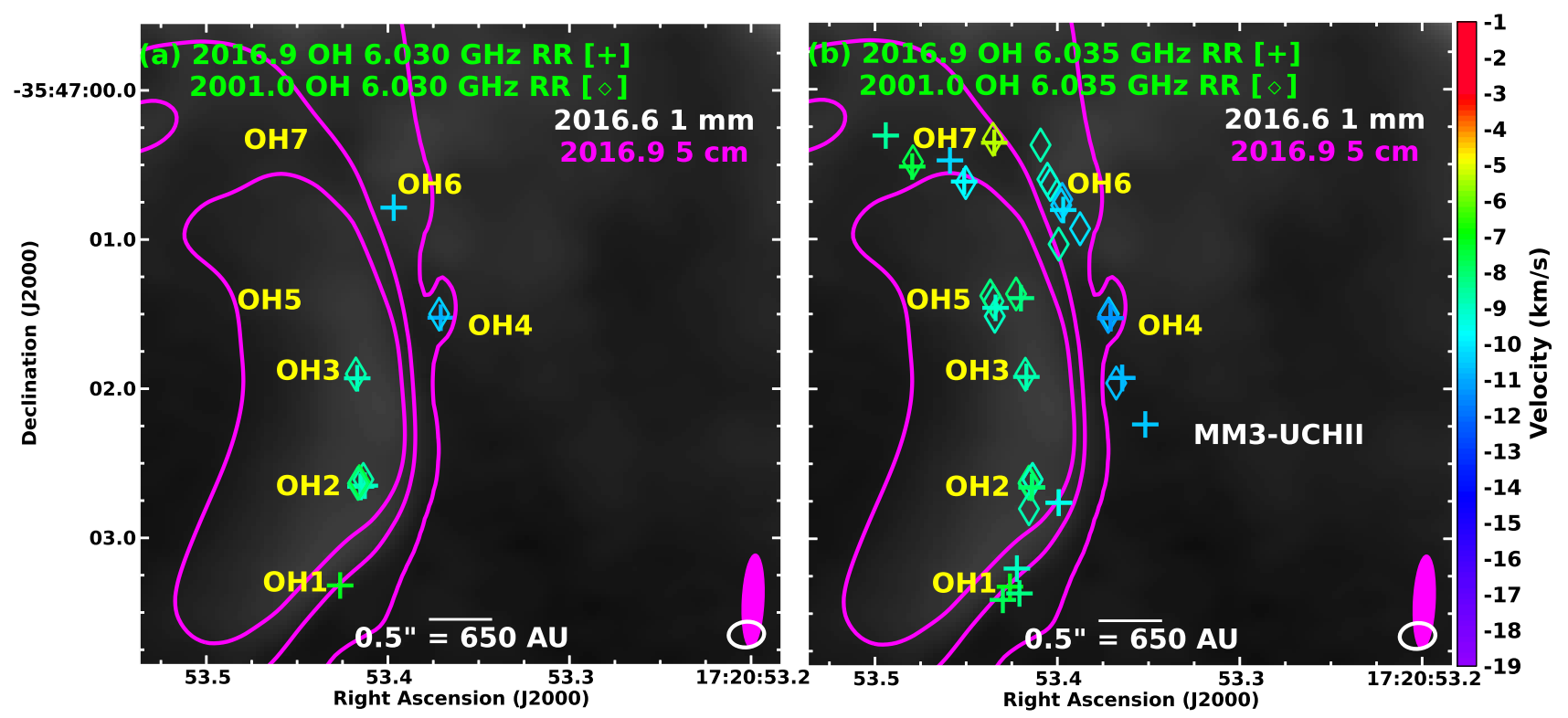

FIG. 5.- The positions of the epoch 2016.9 VLA Zeeman pairs toward the MM3-UCHII region are plotted as crosses. The epoch 2001.0 pairs found by Caswell et al. (2011) are plotted as diamonds, after applying a - $0 . \prime 1$ shift in right ascension. The background image and contours are the same as Fig. 1 .
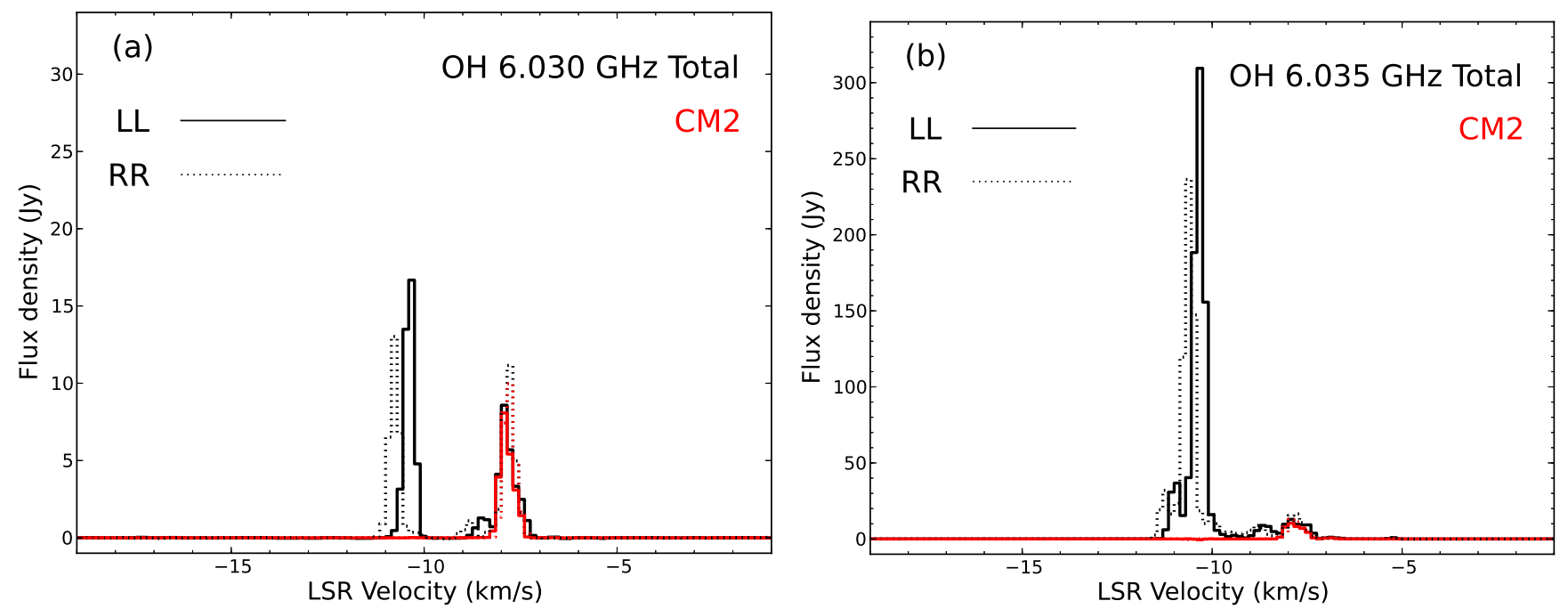

FiG. 6.- Integrated spectra per polarization of the excited-state $\mathrm{OH}$ masers constructed by summing the flux density from all directions (black spectra) and from CM2 only (red spectra): (a) $6.030 \mathrm{GHz}$ line; (b) $6.035 \mathrm{GHz}$ line. Note the factor of 10 change in the y-axis scale between the panels.

the current resolution) are further appended with a letter of the alphabet in increasing velocity order. The lineof-sight magnetic field strength reported in Table 8 was derived using $B_{\text {los }}=\left(V_{\text {center }}(R C P)-V_{\text {center }}(L C P)\right) / Z$ where $Z=0.056 \mathrm{~km} \mathrm{~s}^{-1} \mathrm{mG}^{-1}$ for the $6.035 \mathrm{GHz}$ transition and $0.079 \mathrm{~km} \mathrm{~s}^{-1} \mathrm{mG}^{-1}$ for the $6.030 \mathrm{GHz}$ transition (Yen et al. 1969).

Caswell et al. (2011) also report $B_{\text {los }}$ for these excited $\mathrm{OH}$ transitions from observations using three antennas of the LBA. Although these data have superior angular resolution $\left(0 . .05 \times 00^{\prime \prime} .02\right)$, the absolute position accuracy was estimated to be as poor as \pm 0.2 . In Figure 7 we have over-plotted the Caswell et al. (2011) Zeeman pairs using $\diamond$ symbols, after applying a -0 ". 1 shift in right ascension in order to align them with the (more accurate) VLA Zeeman pairs from Table 8 . From this figure, it is clear that toward the UCHII region many Caswell et al. (2011) Zeeman pairs are still present for both transitions, and with similar velocities. Comparison of the derived $B_{l o s}$ between the present work and Caswell et al. (2011) also yields excellent overall agreement. For example, for spots that are in common between the two epochs at $6.035 \mathrm{GHz}$ in the associations UCHII-OH2, UCHII-OH3, UCHII-OH4, UCHII-OH5, differences in $B_{\text {los }}$ are less than $10 \%$. Only toward UCHII-OH6 are there notable differences in $B_{l o s}$ but this region also showed the largest variation in $B_{l o s}$ in the Caswell et al. (2011) data. For the less prevalent $6.030 \mathrm{GHz}$ transition, the agreement in the derived $B_{\text {los }}$ (for matching spots) with Caswell et al. (2011) is also within $10 \%$. Regarding our VLA data, while most $6.030 \mathrm{GHz}$ spots have derived $B_{\text {los }}$ that agree with the $6.035 \mathrm{GHz}$ measurements, at a few locations 
TABLE 8

Line OF Sight MAGNetic FIELd $\left(B_{l o s}\right)$ DERIVED From OH 6.035 AND $6.030 \mathrm{GHz}$ TRANSitions

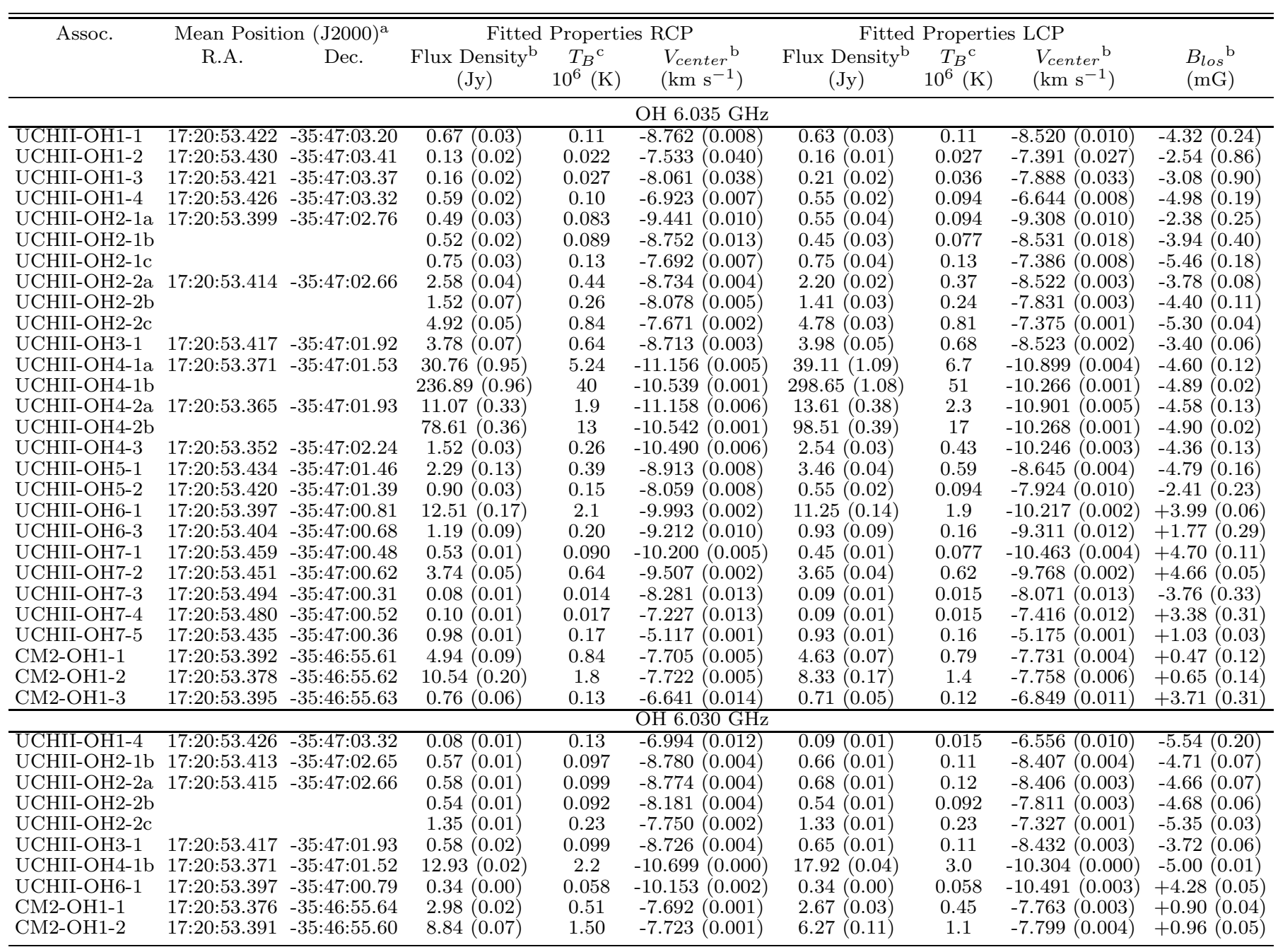

a This is the mean of the intensity-weighted centroids of the associated RCP and LCP spots. In all cases, they are within $1 / 3$ of the synthesized beam minor axis of each other.

b Values in parentheses show the fitted uncertainties.

${ }^{\mathrm{c}}$ Lower limits to the brightness temperature $T_{B}$ using the synthesized beam; the physical sizes of the maser spots are likely to be smaller than the beam $(\sim 520 \mathrm{au})$.

the magnitude of the $6.030 \mathrm{GHz}$-derived field is notably larger (UCHII-OH2-1b, UCHII-OH2-2a, and especially CM2-OH1). This discrepancy may be a result of spectral/spatial blending for the stronger and more confused $6.035 \mathrm{GHz}$ spots.

Additionally, we confirm the finding of Caswell et al. (2011) of a reversal in the sign of $B_{l o s}$ between spots located toward the Southern portions (negative field: UCHII-OH1 to UCHII-OH5) and Northern portions (positive field: UCHII-OH6 and UCHII-OH7) of the $\mathrm{H}$ II region. Interestingly, the most northerly of the spots detected in UCHII-OH7 reveals a second reversal back to negative value (this spot was not detected by Caswell et al. 2011). Toward CM2-OH1, the $B_{l o s}$ is also positive, but somewhat weaker than for the majority of spots toward the $\mathrm{H}$ II region (as discussed previously, this is the first time excited $\mathrm{OH}$ masers have been resolved toward the CM2 region of the protocluster).

\subsection{Excited-state $\mathrm{OH} 4.660 \mathrm{GHz}$ transition}

We did not detect the $4.660 \mathrm{GHz} \mathrm{OH}$ transition to a $3 \sigma$ limit of $10 \mathrm{mJy}^{\mathrm{beam}}{ }^{-1}$. This transition is rarely seen in surveys (see Cohen et al. 1995, for an earlier singledish upper limit of $0.17 \mathrm{Jy}$ ). However, it was detected at a peak flux density of 2.2 Jy in the field of NGC6334I by the HartRAO single dish monitoring during the initial weeks of the flare, making it only the fifth object to have ever exhibited this transition (see MacLeod et al. 2018, and references therein). The emission velocity of the transient $4.660 \mathrm{GHz}$ maser was consistent with the flaring $\mathrm{CH}_{3} \mathrm{OH}$ and $6 \mathrm{GHz} \mathrm{OH}$ masers, suggesting that they were co-located. But because no high-resolution observations exist, we lack definitive proof of this association.

\section{DISCUSSION}

\subsection{Implications from maser pumping schemes}




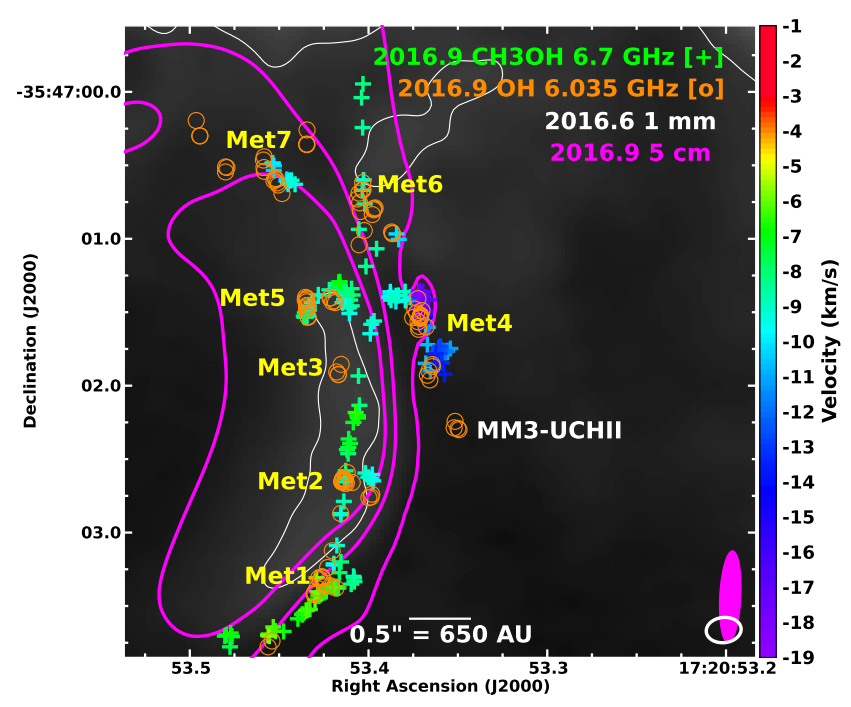

Fig. 7.- Similar to Fig. 2 $\mathrm{d}$ except the RCP OH $6.035 \mathrm{GHz}$ maser spots (o symbols) are overplotted in orange for comparison with the positions of the methanol masers (+ symbols).

The rapid onset of methanol masers on and around MM1 raises the question as to what conditions changed to support the maser inversion. The $6.7 \mathrm{GHz}$ methanol transition is radiatively pumped by mid-infrared photons (Sobolev et al. 1997). The inversion requires $T_{d u s t}$ above $120 \mathrm{~K}$ and can occur across a wide range of gas volume densities (up to about $n=10^{9} \mathrm{~cm}^{-3}$ ) and kinetic temperatures (at least 25 to $250 \mathrm{~K}$ ), as long as the $\mathrm{CH}_{3} \mathrm{OH}$ abundance is greater than $10^{-7}$ (Cragg et al. 2005). Prior to the millimeter continuum outburst, when the dust and gas temperatures were presumably in better equilibrium, the gas temperature provides an estimate for the dust temperature. In the line survey of NGC6334I by Zernickel et al. (2012), the complex organic molecules with the most compact emission exhibited model excitation temperatures of $100 \mathrm{~K}\left(\mathrm{CH}_{3} \mathrm{OH}\right)$ to $150 \mathrm{~K}\left(\mathrm{CH}_{3} \mathrm{CN}\right)$. Furthermore, the abundance of $\mathrm{CH}_{3} \mathrm{OH}$ was modeled as $4.7 \times 10^{-6}$. Following the millimeter continuum outburst, the dust temperature of MM1 inferred directly from the $1.3 \mathrm{~mm}$ continuum brightness temperature exceeds $250 \mathrm{~K}$ toward the central components, and was above $150 \mathrm{~K}$ over a several square arcsecond region (Brogan et al. 2016). Thus, in NGC6334I-MM1, the rapid heating of the dust grains by $\gtrsim 100 \mathrm{~K}$ by the recent accretion outburst can plausibly explain the appearance of $6.7 \mathrm{GHz}$ masers in the gas in the vicinity of the powering source of the outburst, MM1B. Furthermore, the lack of masers toward the central dust peaks (MM1A, $1 \mathrm{~B}$ and 1D) suggests that the density is too high toward these objects to support the inversion, because the dust temperature is surely high enough.

When compared to the ALMA $1 \mathrm{~mm}$ dust distribution (Fig. 2a), the appearance of $6.7 \mathrm{GHz}$ masers to the north, west, and southwest of MM1 may seem perplexing, as the dust emission is relatively weak at all of these locations. In fact, over $80 \%$ of the masers reside outside of the $40 \%$ level of the continuum, and over $50 \%$ reside outside of the $5 \%$ level of the continuum. However, strong thermal molecular line emission is significantly more widespread than the bright dust emission. In Figure 8 we show the peak line intensity for a repre-

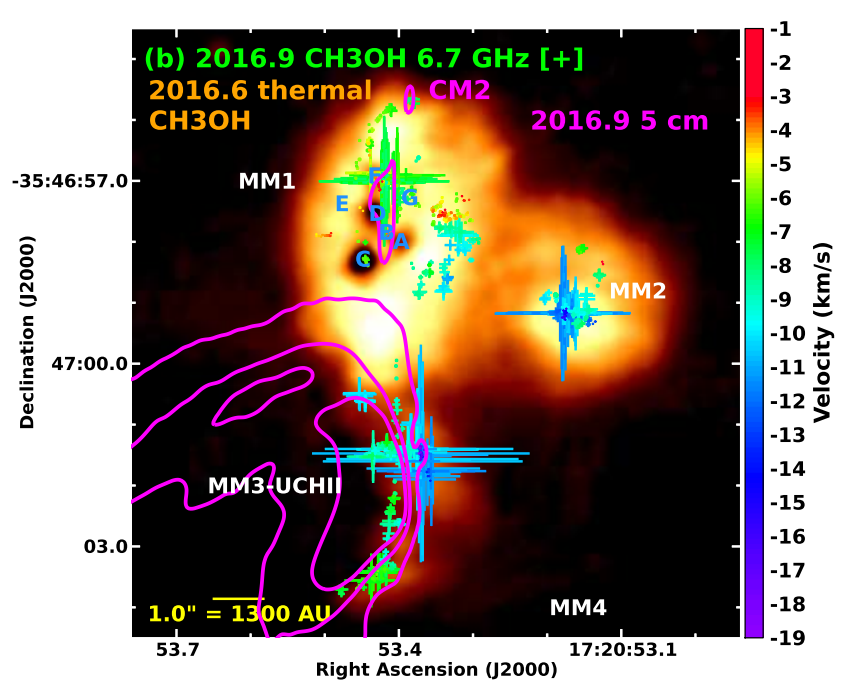

FIG. 8. - The colorscale shows the peak intensity of the thermal methanol transition 11(2)-10(3) with an $E_{\text {Lower }} \widehat{=} 177 \mathrm{~K}$. The same magenta contours, maser spot locations, and velocity color mapping from Fig. 10 are also shown. Blue letters denote the $1.3 \mathrm{~mm}$ continuum peaks in MM1 defined by Brogan et al. (2016).

sentative line from the ALMA $1 \mathrm{~mm}$ data (from whence the dust image also came). The transition shown is

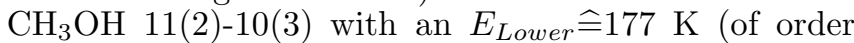
the expected gas kinetic temperature). From comparison of the distribution of $6.7 \mathrm{GHz}$ masers versus the distribution of thermal $\mathrm{CH}_{3} \mathrm{OH}$ (Fig. 8), it is clear that the masers do lie in regions of high molecular column density. The critical density $\left(n_{\text {crit }}\right)$ of the $\mathrm{CH}_{3} \mathrm{OH} 11(2)$ $10(3)$ transition can be computed from the ratio of the Einstein A coefficient $\left(3.46 \times 10^{5} \mathrm{~s}^{-1}\right)$ and the collisional cross section $\left(6 \times 10^{-13} \mathrm{~cm}^{2}\right)$ where the values are taken from Rabli \& Flower (2010) via the LAMDA database (Schöier et al. 2010), yielding $n_{\text {crit }}=6 \times 10^{7} \mathrm{~cm}^{-3}$. While the density at which strong emission can begin to arise from millimeter transitions (the effective excitation density, Shirlev 2015) will be somewhat below $n_{\text {crit }}$, both values are within the required range of $n$ for pumping the maser (Cragg et al. 2005). There is a notable lack of maser emission in the bright thermal gas between MM1 and MM3, suggesting that the gas density is too high here or the dust temperature is too low.

As demonstrated in Figure 7, the $6.7 \mathrm{GHz}$ and excited $\mathrm{OH}$ masers are coincident to within $<0$ "! 1 at a number of locations toward the MM3-UCHII region (see also Caswell 1997), though it is notable that higher angular resolution studies generally find they are not exactly coincident at milliarcsecond scales, e.g. W3 $(\mathrm{OH})$ (Etoka et al. 2005). Figure 9] shows example spectra comparing the velocities of the ionized gas (as traced by the $\mathrm{H} 64 \alpha \mathrm{RRL}$ ), thermal gas (as traced by the $\mathrm{CH}_{3} \mathrm{OH}$ $11(2,10)-10(3,7)$ transition), and $6.7 \mathrm{GHz}$ and $\mathrm{RCP}$ for the $6.035 \mathrm{GHz}$ maser gas toward several of the maser associations coincident with the MM3-UCHII region. Toward all of the maser associations (including those not shown), the velocities of both species of maser emission and of thermal $\mathrm{CH}_{3} \mathrm{OH}$ emission are in excellent agreement, suggesting that these gas components are also at similar locations along the line-of-sight. Toward the maser associations UCHII-Met/OH2, UCHIIMet/OH5, and UCHII-Met/OH7, the neutral gas is no- 

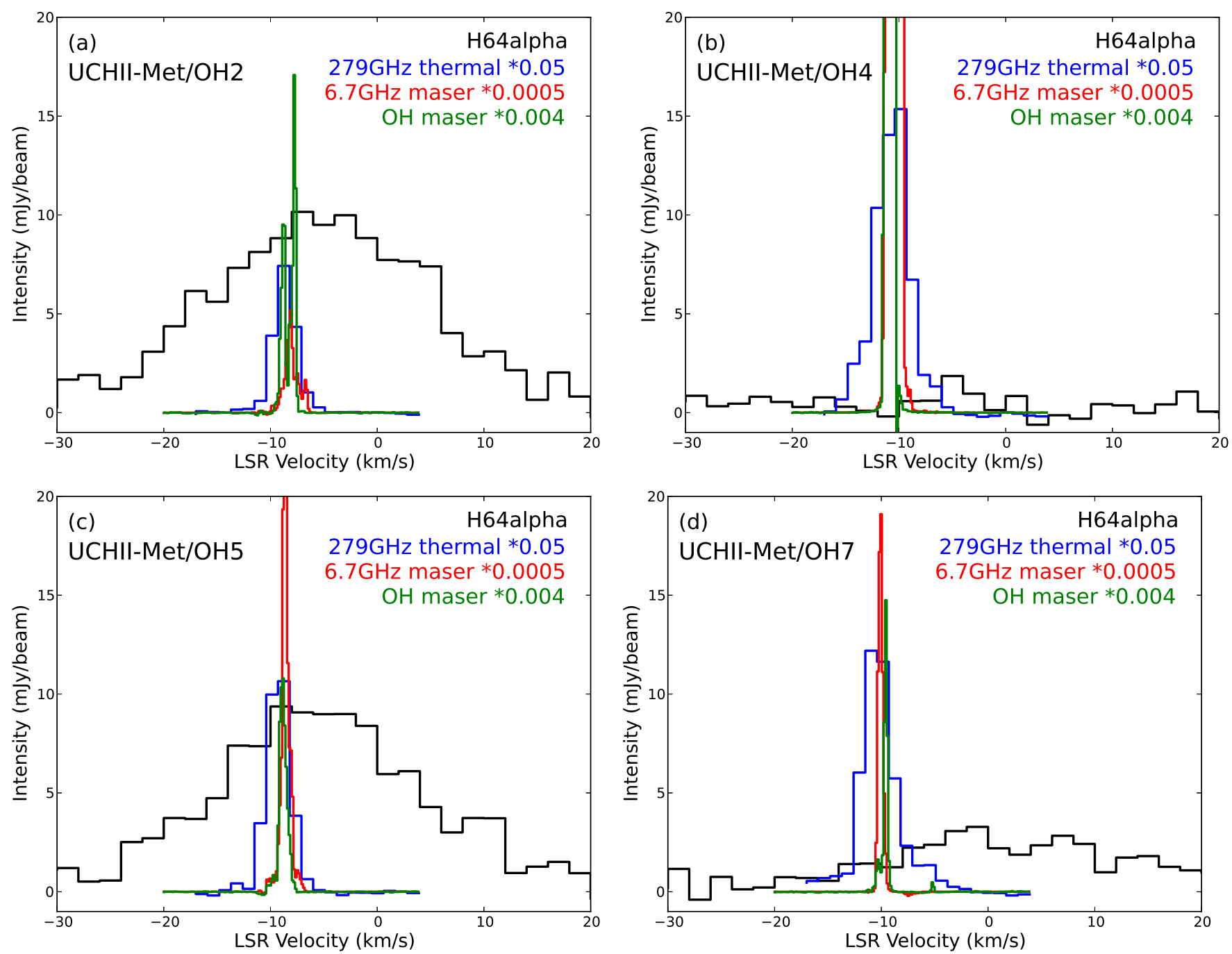

FIG. 9. - Spectral profiles toward several of the maser associations in the MM3-UCHII region are shown. The lines of methanol 6.7 and $279.352 \mathrm{GHz}$ and of $\mathrm{RCP} \mathrm{OH} 6.035 \mathrm{GHz}$ are scaled down in intensity by the factor listed in the legend for ease of comparison to the weak recombination line.

tably blueshifted with respect to the ionized gas. Assuming a typical expanding UCHII region scenario, this suggests that the neutral gas emission originates on the front side of MM3-UCHII. This effect is most dramatic toward the northern UCHII-Met/OH7 association where the neutral gas is blueshifted by $\gtrsim 10 \mathrm{~km} \mathrm{~s}^{-1}$ compared to the ionized gas, such that there is little overlap. It is notable that it is the ionized gas velocity that has changed compared to the other regions, rather than the maser velocities. The significant reddening of the ionized gas velocity toward the northern part of the MM3UCHII region was also observed by de Pree et al. (1995) from VLA observations of the H76 $\alpha$ line. The brightest $6.7 \mathrm{GHz}$ and $\mathrm{OH} 6.035 \mathrm{GHz}$ masers toward the UCHII region are found along its extreme western boundary (UCHII-Met/OH4), coincident with a $\sim 0$ '.25 diameter blister-like bulge in the $5 \mathrm{~cm}$ continuum; unfortunately the $\mathrm{H} 64 \alpha$ line was not detected at this location, so the relationship between the masers and ionized gas cannot be determined. The RRL was not detected toward the CM2 region (nor toward MM1), though as for the UCHII region, the positions and velocities of the $\mathrm{CM} 26.7 \mathrm{GHz}$ and $\mathrm{OH} 6.035 \mathrm{GHz}$ masers are similar, and their peak velocities match the thermal methanol that is present there.

Excited state $\mathrm{OH}$ masers are efficiently pumped for a lower range of kinetic (gas) temperatures than the $6.7 \mathrm{GHz} \mathrm{CH}_{3} \mathrm{OH}$ transition: $25-70 \mathrm{~K}$ (see for example Cragg et al. 2002). Therefore, the excellent kinematic agreement between the two maser species suggests that the molecular gas toward the MM3-UCHII region is cooler than toward MM1 (where excited OH masers are not detected). The maser models of Cragg et al. (2002, 2005) also suggest that the excited 6.030 and $6.035 \mathrm{GHz}$ $\mathrm{OH}$ transitions are only effectively pumped toward the upper end of the density range predicted for $6.7 \mathrm{GHz}$ $\mathrm{CH}_{3} \mathrm{OH}$ masers $\left(10^{6.5}\right.$ to $10^{8.3} \mathrm{~cm}^{-3}$ compared to $10^{4}$ to $\left.10^{8.3} \mathrm{~cm}^{-3}\right)$. Interestingly, the Cragg et al. (2002) models also suggest that the ratio of $6.035 / 6.030 \mathrm{GHz} \mathrm{OH}$ maser brightness temperature is minimized, and indeed becomes nearly equal, for the highest densities, which may explain the anomalous ratio observed toward CM2 if the gas density is higher there. Future multi-transition analysis of the thermal molecular gas throughout the pro- 
tocluster will help to verify that the observed physical conditions meet the predictions of maser pump models.

The range of the magnetic field strengths we measure in excited $\mathrm{OH}(0.5-5 \mathrm{mG})$ is very similar to that found in other high-mass star forming regions observed interferometrically in these transitions (e.g., W51 Main, W3 (OH), and ON1, Etoka et al. 2012; Fish \& Siouwerman 2007; Green et al. 2007). These values are about five times higher than those measured in single-dish $\mathrm{CN}$ observations of massive star forming regions (Falgarone et al. 2008), however those observations sample gas at larger scales and hence lower average density $\left(1-20 \times 10^{5} \mathrm{~cm}^{-3}\right)$. Because magnetic field strength is expected to scale with the square root of density (Crutcher 1999), the excited OH masers apparently arise from gas at densities that are $\sim 25$ times higher, i.e. $0.2-5 \times 10^{7} \mathrm{~cm}^{-3}$. Radiative transfer modeling of these transitions predict that $\mathrm{OH}$ densities of $\sim 10 \mathrm{~cm}^{-3}$ are required to pump these masers, which are consistent with $\mathrm{H}_{2}$ densities of $5 \times 10^{7} \mathrm{~cm}^{-3}$ using the nominal $\mathrm{OH}$ abundance of $2 \times 10^{-7}$ (Etoka et al. 2012). Thus, the density implied by the measured excited $\mathrm{OH}$ field strengths is in good agreement with the value required for the thermal methanol emission and the pumping models for excited $\mathrm{OH}$ maser emission.

\subsection{Spatial and kinematic structures}

The sudden appearance of strong $6.7 \mathrm{GHz}$ methanol masers on and around MM1 demonstrates an important new characteristic of the Class II maser phenomenon, which has presented an ongoing enigma to observers. While Class I methanol masers (Leurini et al. 2016) trace ambient-velocity shocked gas at the interfaces of outflow structures and can be located far from the driving source (Cyganowski et al. 2009; Rodríguez-Garza et al. 2017), Class II methanol masers are always found in close proximity to other tracers of massive protostars such as submillimeter continuum (Urquhart et al. 2013), midinfrared continuum (Bartkiewicz et al.|2014), or hot core molecular gas (Chibueze et al. 2017). An early imaging survey with the ATCA at $\sim 1^{\prime \prime}$ resolution found linear distributions of fitted spots across (up to) a few synthesized beams, which were interpreted as a indicator of either edge-on protoplanetary disks or collimated jets (Norris et al. 1993). Interestingly, two of the 10 so-called "linear" sources were the two concentrations of emission in NGC6334F (the previously-known masers associated with MM2 and MM3). Although only half of the cases exhibited a corresponding velocity gradient, the edgeon disk interpretation became preferred in the literature (Norris et al. 1998).

However, the disk hypothesis was subsequently tested by searching for outflow lobes perpendicular to the linear structures by imaging the shock-tracing near-infrared $\mathrm{H}_{2}$ line in 28 such maser sources (De Buizer 2003). Only two sources showed outflowing gas in the perpendicular direction, while in contrast $80 \%$ of the cases (with radiatively excited $\mathrm{H}_{2}$ ) showed emission parallel to the maser axis, instead suggesting an association with the outflow. Further evidence for an association between $6.7 \mathrm{GHz}$ masers and outflows has come from other studies of individual sources (e.g. NGC7538 IRS1, De Buizer \& Minier 2005). Nevertheless, the disk interpretation continues to be invoked to explain recent observations of individual mas- sive protostars, such as Cepheus A HW2 (Sanna et al. 2017) and G353.273+0.641 (Motogi et al. 2017); however in these cases, the proposed disks have an inclined or face-on geometry. In one case, proper motion studies suggest masers reside in both the disk and outflow (IRAS 20126+4104, Moscadelli et al. 2011). Recently, a VLBI imaging survey of 31 methanol masers identified a new class of structure: a ring-like distribution of spots in $29 \%$ of the sample (Bartkiewicz et al. 2009), with the most striking example being G23.6570.127 (Bartkiewicz et al. 2005). With typical radii of $\lesssim 0^{\prime \prime} 1$, these rings are smaller than the scales probed by the VLA or ATCA. A follow-up study in the near-IR and mid-IR again found no evidence to support the idea that these rings trace protostellar disks (De Buizer et al. 2012). Proper motion studies show that the ring of masers in G23.657-0.127 is expanding (Szymczak et al. 2017) and while the interpretation is still unclear, it may be the result of a disk wind (Bartkiewicz et al. 2017).

Before interpreting the location of the flaring masers, we summarize the current prior knowledge of star formation in MM1. The original ALMA observations resolved MM1 into multiple dust continuum components, which we modeled as seven two-dimensional Gaussian sources at $1.3 \mathrm{~mm}$ (A-F, Brogan et al. 2016). Since the molecular hot core emission encompasses all these components, each one should be considered as a candidate protostar. However, the water maser emission from MM1 (VLA epoch 2011.7) was associated with only the two brightest components, B and D. The small upper limit to the size of B at $7 \mathrm{~mm}\left(<0^{\prime \prime} .18 \sim 230 \mathrm{au}\right)$ led us to model its spectral energy distribution (SED) as a dense hypercompact HII (HCHII) region, while the SED and larger size of $\mathrm{D}$ were more consistent with emission from a jet. A single component fit to the elongated $5 \mathrm{~cm}$ emission feature (epoch 2011.4) peaks near D, but extends down to $\mathrm{B}$ and up to an area between $\mathrm{F}$ and $\mathrm{G}$. We interpreted this emission as a jet, but its origin and whether it is symmetric with respect to its driving source (D) or more asymmetric (B) were unclear. Besides B and D, the only other source detected at $7 \mathrm{~mm}$ is $\mathrm{F}$, whose SED is consistent with dust emission alone, suggesting an earlier stage protostar. The other $5 \mathrm{~cm}$ source, CM2, was enigmatic as its emission is non-thermal and it exhibits the brightest water maser emission yet no compact dust emission.

The distribution of the new flaring masers in MM1 exhibits a variety of shapes. There are a few linear arrangements of spots, perhaps the most striking of which is the "V"-shaped structure of masers between MM1F and $1 \mathrm{G}$ (Fig. 2b). Because this structure lies at the northern end of the $5 \mathrm{~cm}$ continuum emission, and along an axis parallel to this emission that intersects MM1B, it may indicate an association between the masers and a jet originating from the HCHII region MM1B. This axis also passes close to the non-thermal radio source CM2, suggesting that it traces a shock against the ambient medium, as proposed for the non-thermal component in the $\mathrm{HH} 80$ 81 jet (Rodríguez-Kamenetzky et al. 2017). How this jet relates to the large scale $\mathrm{NE} / \mathrm{SW}$ bipolar outflow remains uncertain from these data, but we note a similarity to the G5.89-0.39 protocluster, which exhibits a young compact outflow and an older larger scale outflow at very different position angles (Hunter et al. 2008; cf. also Puga et al. 2006). While it is also unclear if the maser spots near 
MM1F are associated with an early stage protostar at that location, the thread of spots that continues northward (CM2-Met2) curving toward the non-thermal continuum source CM2 (Fig. 2a) supports an association with the jet. The spots north of MM1G partially form a similar structure west of the jet, which suggests that both sets of masers follow the walls of an outflow cavity. Proper motion studies with VLBI would be helpful to test this hypothesis (Chibueze et al., in preparation).

Shorter linear collections of maser spots also appear in MM1-MetC and MM1-Met3. The dust emission from MM1C indicates there may be a protostar associated with MM1-MetC, however the velocity range of these spots is rather small $\left(2.7 \mathrm{~km} \mathrm{~s}^{-1}\right)$ and lacks a systematic gradient. MM1-Met3 does show an east/west velocity gradient, but the velocity range is even smaller $\left(1.3 \mathrm{~km} \mathrm{~s}^{-1}\right)$ and there is no evidence for a protostar as a point source in the dust emission. Linear features with such narrow velocity ranges are similar to those seen in LBA images of five other HMYSOs by Dodson et al. (2004), who conclude that they trace planar shocks propagating perpendicular to the line of sight rather than disks. Similar to MM1-Met3, the copious maser spots of MM1-Met2 have no direct association with a protostar, since there are no millimeter point sources in that area to very sensitive levels (Brogan et al. 2016). Instead, these maser spots look more like they arise from a collection of filaments, similar to those seen in $\mathrm{W} 3 \mathrm{OH}$ where masers trace filaments extending up to $3100 \mathrm{au}$ (Harvey-Smith \& Cohen 2006). It would appear that the presence of maser filaments can occur over a broad range in evolutionary state of massive protostars since the masers in MM3-UCHII (Met1 through Met7) appear to delineate a filament nearly $5000 \mathrm{au}$ in length (Fig. 7).

In the other HMYSO hosting methanol masers in this protocluster, MM2, we see a similar dichotomy as in MM1, with the spots of MM2-Met1 appearing close to the protostar(s) traced by the continuum sources MM2A and 2B, while those of MM2-Met2 appear further afield $(>1000 \mathrm{au})$. So the primary conclusion we draw from this outburst is that although some $6.7 \mathrm{GHz}$ masers do reside close to massive protostars (within $500 \mathrm{au}$ ), significant amounts of maser emission can occur in the gas further away as long as the radiative pumping from nearby protostars is sufficient in those locations. The presence of outflow cavities driven by a central protostar likely creates propagation paths for infrared photons that increase the range of the required radiative pumping to these larger distances. Indeed, the brightest masers can lie along these paths, as evidenced by MM1-Met1, which lies $\approx 1000$ au from the outburst source MM1B. This result is strikingly similar to the other recent flaring maser source S255 NIRS3, in which the brightest maser emission also lies 500-1000 au from the flaring protostar (Moscadelli et al. 2017).

\subsection{Origin of the 1999 methanol maser flare}

Goedhart et al. (2004) presented single-dish monitoring data of the $6.7 \mathrm{GHz}$ line from 28 February 1999 to 27 March 2003 and noted a large flare in the velocity component at $-5.88 \mathrm{~km} \mathrm{~s}^{-1}$. Re-analysis of these data (MacLeod et al. 2018) indicates that the flare peaked on 19 November 1999 (epoch 1999.88) at a velocity of $-5.99 \mathrm{~km} \mathrm{~s}^{-1}$ with a peak flux density 230 times higher than the pre-burst value of $1.6 \pm 0.4 \mathrm{Jy}$. While there are no published interferometric maps from that epoch, we can examine the location of the emission at this velocity in our recent epoch 2016.9 VLA data. In the $-5.90 \mathrm{~km} \mathrm{~s}^{-1}$ channel, we find a total flux density of $6.41 \mathrm{Jy}$, only $10 \%$ of which originates from any of the UCHII associations (only UCHII-Met1). The majority (5.41 Jy) originates from MM1, and the rest $(0.34 \mathrm{Jy})$ from CM2-Met2. Since the rest of the emission (0.70 Jy, from UCHII-Met1 and MM2-Met2) is consistent with the quiescent single-dish value found after the 1999 flare $(0.55 \pm 0.20 \mathrm{Jy})$, it is quite plausible that this flare could have also originated entirely from MM1 and CM2. This result supports the hypothesis of MacLeod et al. (2018) that the 1999 and 2015 events arise from the same physical location and are due to a common repeating mechanism such as the decaying orbit of a binary system embedded in dense gas (Stahler 2010). Continued long-term monitoring of the maser emission is essential to test this scenario. It is particularly important to measure how long the current maser flare lasts compared to the more limited 1999 event. If this flare persists for many years, it increases the likelihood that a significant population of the masers found in surveys arise from protostars that have recently undergone a large accretion outburst.

\section{CONCLUSIONS}

The recent extraordinary outburst in the massive protostellar cluster NGC6334I provides an unprecedented window into the central environment of a massive starforming region. We have obtained high resolution ( 500 au) VLA images of multiple maser transitions which reveal the first appearance of Class II methanol maser emission toward the MM1 protostellar system in over 30 years of past observations. The $6.7 \mathrm{GHz}$ masers are distributed toward particular parts of MM1 (including MM1C, F and G), on its northern and western peripheries, and toward the non-thermal radio source CM2. The masers are strikingly absent from the brightest millimeter components (MM1A, B and D). We find that current models of maser pumping can explain the general location of the masers in the context of the recent luminous outburst from MM1B. The spatio-kinematic structures traced by the $6.7 \mathrm{GHz}$ masers are varied but support the idea that while some masers do reside close to massive protostars (within $500 \mathrm{au}$ ) traced by compact dust emission, significant maser action can also occur in more extended areas associated with strong thermal gas emission. Also, the presence of jets and outflow cavities driven by a central protostar increases the range of infrared pumping photons, allowing strong masers to appear further away (> $1000 \mathrm{au})$.

Our simultaneous observations of two of the $6 \mathrm{GHz}$ excited state $\mathrm{OH}$ transitions reveal no emission toward MM1, but we do detect a strong new maser at $-7.7 \mathrm{~km} \mathrm{~s}^{-1}$ arising from the non-thermal radio source CM2. Not previously detected by the LBA, it was apparently excited by the recent millimeter outburst. By analyzing the RCP and LCP data cubes, we identify several Zeeman pairs to measure the line of sight magnetic field in the UCHII region and CM2. We confirm the field strengths and the reversal in field direction across the UCHII region identified by Caswell et al. (2011). The magnetic field toward CM2 is significantly weaker than 
toward the majority of positions across the UCHII region, and the flux ratio between the 6.035 and $6.030 \mathrm{GHz}$ lines is anomalous, with the lines being comparable in strength toward CM2. OH maser pumping models suggest that the gas density is significantly higher toward CM2, consistent with compression of gas by the propagation of the jet from MM1B.

Future high resolution observations of the $\mathrm{CH}_{3} \mathrm{OH}$ and $\mathrm{OH}$ masers will be essential to obtain proper motions of the new features in order to determine which ones may be tracing structures associated with an outflow versus those that may be bound to a protostar. Comparison with thermal gas outflow tracers imaged by ALMA will then be possible. Continued single dish monitoring is also critical to measure the lifetime of the current maser flare and to search for future events in order to test the hypothesis that a periodic phenomenon such as an eccentric protostellar binary orbit may be responsible for repeating accretion outbursts.

We thank the anonymous referee for a thorough review which has improved the manuscript. The
National Radio Astronomy Observatory is a facility of the National Science Foundation operated under agreement by the Associated Universities, Inc. This paper makes use of the following ALMA data: ADS/JAO.ALMA\#2015.A.00022.T. ALMA is a partnership of ESO (representing its member states), NSF (USA) and NINS (Japan), together with NRC (Canada) and NSC and ASIAA (Taiwan) and KASI (Republic of Korea), in cooperation with the Republic of Chile. The Joint ALMA Observatory is operated by ESO, AUI/NRAO and NAOJ. This research made use of NASA's Astrophysics Data System Bibliographic Services, the SIMBAD database operated at CDS, Strasbourg, France, Astropy, a community-developed core Python package for Astronomy (Astropy Collaboration et al. 2013), and APLpy, an open-source plotting package for Python hosted at http://aplpy.github.com T. Hirota is supported by the MEXT/JSPS KAKENHI grant No. 17 K05398. C.J. Cyganowski acknowledges support from the STFC (grant number ST/M001296/1).

Facilities: VLA, ALMA.
Abraham, Z., Cohen, N. L., Opher, R., Raffaelli, J. C., \& Zisk, S. H. 1981, A\&A, 100, L10

Astropy Collaboration, Robitaille, T. P., Tollerud, E. J., et al. 2013, A\&A, 558, A33

Avison, A., Quinn, L. J., Fuller, G. A., et al. 2016, MNRAS, 461, 136

Bartkiewicz, A., et al. 2017, IAU Symp. 336

Bartkiewicz, A., Szymczak, M., \& van Langevelde, H. J. 2014 A\&A, 564, A110

Bartkiewicz, A., Szymczak, M., van Langevelde, H. J., Richards, A. M. S., \& Pihlström, Y. M. 2009, A\&A, 502, 155

Bartkiewicz, A., Szymczak, M., \& van Langevelde, H. J. 2005, A\&A, 442, L61

Beuther, H., Walsh, A. J., Thorwirth, S., et al. 2008, A\&A, 481, 169

Beuther, H., Walsh, A. J., Thorwirth, S., et al. 2007, A\&A, 466, 989

Brogan, C. L., Hunter, T. R., Cyganowski, C. J., et al. 2016, ApJ 832,187

Caratti o Garatti, A., Stecklum, B., Garcia Lopez, R., et al. 2017, Nature Physics, 13, 276

Caswell, J. L., \& Vaile, R. A. 1995, MNRAS, 273, 328

Caswell, J. L. 1997, MNRAS, 289, 203

Caswell, J. L., Kramer, B. H., \& Reynolds, J. E. 2011, MNRAS, 414,1914

Caswell, J. L. 2003, MNRAS, 341, 551

Chibueze, J. O., Csengeri, T., Tatematsu, K., et al. 2017, ApJ, 836,59

Chibueze, J. O., Omodaka, T., Handa, T., et al. 2014, ApJ, 784, 114

Cohen, R. J., Masheder, M. R. W., \& Caswell, J. L. 1995, MNRAS, 274, 808

Contreras Peña, C., Lucas, P. W., Minniti, D., et al. 2017, MNRAS, 465, 3011

Cragg, D. M., Sobolev, A. M., \& Godfrey, P. D. 2005, MNRAS, 360,533

Cragg, D. M., Sobolev, A. M., \& Godfrey, P. D. 2002, MNRAS, 331,521

Crutcher, R. M. 1999, ApJ, 520, 706

Cyganowski, C. J., Brogan, C. L., Hunter, T. R., \& Churchwell, E. 2009, ApJ, 702, 1615

De Buizer, J. M., Bartkiewicz, A., \& Szymczak, M. 2012, ApJ, 754,149

De Buizer, J. M.. \& Minier, V. 2005, ApJ, 628, L151

De Buizer, J. M. 2003, MNRAS, 341, 277

De Buizer, J. M., Radomski, J. T., Piña, R. K., \& Telesco, C. M. 2002, ApJ, 580, 305

de Pree, C. G., Rodriguez, L. F., Dickel, H. R., \& Goss, W. M. 1995, ApJ, 447, 220

Dodson, R., \& Moriarty, C. D. 2012, MNRAS, 421, 2395

Dodson, R., Ojha, R., \& Ellingsen, S. P. 2004, MNRAS, 351, 779

Elitzur, M. 1992, ARA\&A, 30, 75

Ellerbroek, L. E., Kaper, L., Bik, A., et al. 2011, ApJ, 732, L9
Ellingsen, S. 1996, Ph.D. Thesis, University of Tasmania

Ellingsen, S. P., Norris, R. P., Diamond, P. J., et al. 1996, Proc. of Third Asia-Pacific Telescope Workshop, arXiv:astro-ph/9604024

Etoka, S., Gray, M. D., \& Fuller, G. A. 2012, MNRAS, 423, 647

Etoka, S., Cohen, R. J., \& Gray, M. D. 2005, MNRAS, 360, 1162

Evans, N. J., II, Dunham, M. M., Jørgensen, J. K., et al. 2009,

ApJS, 181, 321-350

Falgarone, E., Troland, T. H., Crutcher, R. M., \& Paubert, G. 2008, A\&A, 487, 247

Fish, V. L., \& Sjouwerman, L. O. 2007, ApJ, 668, 331

Fujisawa, K., Yonekura, Y., Sugiyama, K., et al. 2015, The Astronomer's Telegram, 8286

Fujisawa, K., Sugiyama, K., Aoki, N., et al. 2012, PASJ, 64, 17

Gardner, F. F., Ribes, J. C., \& Goss, W. M. 1970,

Astrophys. Lett., 7, 51

Goedhart, S., Gaylard, M. J., \& van der Walt, D. J. 2004 MNRAS, 355, 553

Gray, M. D., Baudry, A., Richards, A. M. S., et al. 2016, MNRAS, 456, 374

Green, J. A., Caswell, J. L., \& McClure-Griffiths, N. M. 2015, MNRAS, 451, 74

Green, J. A., Richards, A. M. S., Vlemmings, W. H. T., Diamond, P., \& Cohen, R. J. 2007, MNRAS, 382, 770

Harvey-Smith, L., \& Cohen, R. J. 2006, MNRAS, 371, 1550

Herbig, G. H. 1977, ApJ, 217, 693

Herbig, G. H. 1989, European Southern Observatory Conference and Workshop Proceedings, 33, 233

Hirota, T., Tsuboi, M., Kurono, Y., et al. 2014, PASJ, 66, 106

Honma, M., Yoon, K. C., Bushimata, T., et al. 2004, PASJ, 56, L15

Hunter, T. R., Brogan, C. L., MacLeod, G., et al. 2017, ApJ, 837, L29

Hunter, T. R., Brogan, C. L., Indebetouw, R., \& Cyganowski, C. J. 2008, ApJ, 680, 1271

Hunter, T. R., Brogan, C. L., Megeath, et al., 2006, ApJ, 649, 888

Kenyon, S. J., Hartmann, L. W., Strom, K. M., \& Strom, S. E. 1990, AJ, 99, 869

Knowles, S. H., Caswell, J. L., \& Goss, W. M. 1976, MNRAS, 175,537

Knowles, S. H., Johnson, K. J., Moran, J. M., \& Ball, J. A. 1973, ApJ, 180, L117

Krishnan, V., Ellingsen, S. P., Voronkov, M. A., \& Breen, S. L. 2013, MNRAS, 433, 3346

Kumar, M. S. N., Contreras Peña, C., Lucas, P. W., \& Thompson, M. A. 2016, ApJ, 833, 24

Lekht, E. E., Pashchenko, M. I., Rudnitskij, G. M., \& Tolmachev, A. M. 2017, Astronomy Reports, submitted, arXiv:1709.08197

Leurini, S., Menten, K. M., \& Walmsley, C. M. 2016, A\&A, 592, A31

Leurini, S., Schilke, P., Parise, B., et al., 2006, A\&A, 454, L83

Liljeström, T., \& Gwinn, C. R. 2000, ApJ, 534, 781 
Lucas, P. W., Smith, L. C., Contreras Pena, C., et al. 2017, MNRAS, 472, 2990

Lucas, P. W., Hoare, M. G., Longmore, A., et al. 2008, MNRAS, 391,136

Macleod, G. et al., 2018, submitted to MNRAS

Mairs, S., Lane, J., Johnstone, D., et al. 2017, ApJ, 843, 55

McCutcheon, W. H., Sandell, G., Matthews, H. E., et al. 2000, MNRAS, 316, 152

McGuire, B. A., et al. 2017, ApJ, 851, L46

Meyer, D. M.-A., Vorobyov, E. I., Kuiper, R., \& Kley, W. 2017, MNRAS, 464, L90

Minier, V., Booth, R. S., \& Conway, J. E. 2002, A\&A, 383, 614

Minniti, D., Lucas, P. W., Emerson, J. P., et al. 2010, New Astronomy, 15, 433

Mitchell, G. F., Maillard, J.-P., \& Hasegawa, T. I. 1991, ApJ, 371,342

Moscadelli, L., Sanna, A., Goddi, C., et al. 2017, A\&A, 600, L8

Moscadelli, L., Cesaroni, R., Rioja, M. J., Dodson, R., \& Reid, M. J. 2011, A\&A, 526, A66

Motogi, K., Hirota, T., Sorai, K., et al. 2017, ApJ, 849, 23

Norris, R. P., Caswell, J. L., Wellington, K. J., McCutcheon, W. H., \& Reynolds, J. E. 1988, Nature, 335, 149

Norris, R. P., Whiteoak, J. B., Caswell, J. L., Wieringa, M. H., \& Gough, R. G. 1993, ApJ, 412, 222

Norris, R. P., Byleveld, S. E., Diamond, P. J., et al. 1998, ApJ, 508,275

Offner, S. S. R., \& McKee, C. F. 2011, ApJ, 736, 53

Omodaka, T., Maeda, T., Miyoshi, M., et al. 1999, PASJ, 51, 333

Persi, P., Tapia, M., Felli, M., Lagage, P. O., \& Ferrari-Toniolo, M. 1998, A\&A, 336, 1024

Puga, E., Feldt, M., Alvarez, C., et al. 2006, ApJ, 641, 373

Qiu, K., Wyrowski, F., Menten, K. M., et al. 2011, ApJ, 743, L25

Qiu, K., \& Zhang, Q. 2009, ApJ, 702, L66

Rabli, D., \& Flower, D. R. 2010, MNRAS, 406, 95

Reid, M. J., Menten, K. M., Brunthaler, A., et al. 2014, ApJ, 783, 130

Reid, M. J., \& Moran, J. M. 1981, ARA\&A, 19, 231

Ridge, N. A., \& Moore, T. J. T. 2001, A\&A, 378, 495

Rodríguez-Kamenetzky, A., Carrasco-González, C., Araudo, A., et al. 2017, ApJ, 851, 16
Rodríguez-Garza, C. B., Kurtz, S. E., Gómez-Ruiz, A. I., et al. 2017, Ap.JS, 233, 4

Rodriguez, L. F., Canto, J., \& Moran, J. M. 1982, ApJ, 255, 103

Safron, E. J., Fischer, W. J., Megeath, S. T., et al. 2015, ApJ, $800, \mathrm{~L} 5$

Sanna, A., Moscadelli, L., Surcis, G., et al. 2017, A\&A, 603, A94

Sault, R.J. \& Sowinski, K. 2013, EVLA Memo 169

Schöier, F., van der Tak, F., van Dishoeck, E., \& Black, J. 2010, Astrophysics Source Code Library, ascl:1010.077

Shirley, Y. L. 2015, PASP, 127, 299

Sobolev, A. M., Cragg, D. M., \& Godfrey, P. D. 1997, A\&A, 324, 211

Stahler, S. W. 2010, MNRAS, 402, 1758

Stutz, A. M., Tobin, J. J., Stanke, T., et al. 2013, ApJ, 767, 36

Szymczak, M. et al., 2017, MNRAS, submitted

Tapia, M., Roth, M., \& Persi, P. 2015, MNRAS, 446, 4088

Tapia, M., Persi, P., \& Roth, M. 1996, A\&A, 316, 102

Thi, W.-F., van Dishoeck, E. F., Pontoppidan, K. M., \& Dartois, E. 2010, MNRAS, 406, 1409

Tolmachev, A. 2011, The Astronomer's Telegram, 3177

Urquhart, J. S., Moore, T. J. T., Schuller, F., et al. 2013, MNRAS, 431, 1752

Volvach, A. E., Volvach, L. N., MacLeod, G., et al. 2017b, The Astronomer's Telegram, 10853

Volvach, A. E., Volvach, L. N., MacLeod, G., et al. 2017a, The Astronomer's Telegram, 10728

Walsh, A. J., Burton, M. G., Hyland, A. R., \& Robinson, G. 1999, MNRAS, 309, 905

Walsh, A. J., Burton, M. G., Hyland, A. R., \& Robinson, G. 1998, MNRAS, 301, 640

Willis, S., Marengo, M., Allen, L., et al. 2013, ApJ, 778, 96

Wu, Y. W., Sato, M., Reid, M. J., et al. 2014, A\&A, 566, A17

Yen, J. L., Zuckerman, B., Palmer, P., \& Penfield, H. 1969, ApJ, 156, L27

Yoo, H., Lee, J.-E., Mairs, S., et al. 2017, ApJ, 849, 69

Zernickel, A., Schilke, P., Schmiedeke, A., et al. 2012, A\&A, 546, A87

Zhang, Q., Qiu, K., Girart, J. M., et al. 2014, ApJ, 792, 116

Zuckerman, B., Yen, J. L., Gottlieb, C. A., \& Palmer, P. 1972, ApJ, 177, 59 